\title{
Development of Simple Alcohols as Nucleophiles for Palladium-catalyzed DYKAT reactions: Total Synthesis of (+)-Hippospongic Acid A
}

\author{
Barry M. Trost,* Michelle R. Machacek, and Hong C. Tsui \\ Department of Chemistry, Stanford University, Stanford, CA 94305
}

\section{Experimental Section}

General Methods. All reactions were performed under an atmosphere of dry argon in flame-dried glassware unless otherwise indicated. Solvents were distilled under an atmosphere of argon before use and transferred via an oven dried syringe or cannula. Diisopropylamine, pyridine, and triethylamine were distilled from calcium hydride. Methanol was distilled from magnsium methoxide. Dichloromethane, 1,4-dioxane, tetrahyrofuran, and toluene were purified by an alumina column purification system. Bis[ $\pi$-allylpalladium chloride] was prepared by the procedure of Shaw. ${ }^{1}$ Tris(dibenzylideneacteone)dipalladium monochloroform complex, $\mathrm{Pd}_{2} \mathrm{dba}_{3} \cdot \mathrm{CHCl}_{3}$, was prepared by the known method of Ibers. ${ }^{2}$ Chiral ligands were prepared by the method of Trost. $^{3}$ All other reagents were obtained from Aldrich, Fluka, or Acros unless otherwise noted.

Flash chromatography was performed with EM Science silica gel (0.040-0.063 $\mu \mathrm{m}$ grade) according to the procedure of Still. ${ }^{4}$ Solvents for chromatography are listed as volume/volume ratios. Analytical thin layer chromatography was performed using $0.2 \mathrm{~mm}$ coated commercial silica gel plates (E. Merck, DC-Plastikfolien, Kieselgel $60 \mathrm{~F}_{254}$ ).

Infrared spectra were recorded on a Perkin Elmer Paragon 500 FT-IR spectophotometer using $0.1 \mathrm{~mm}$ path lenth sodium chloride cavity cells or sodium chloride plates. Absorbance frequencies are recorded in reciprocal centimeters $\left(\mathrm{cm}^{-1}\right)$. Elemental analyses were performed by M-H-W Laboratories, Phoenix, Arizona. High resolution mass spectra (HRMS) were obtained from the Mass Spectrometry Resource, School of Pharmacy, University of California-San Francisco on a Kratos MS9 spectrometer. HRMS data are reported as $\mathrm{m} / \mathrm{e}$ (relative intensity), with accurate mass reported for the molecular ion $\left(\mathrm{M}^{+}\right)$or suitable fragments.

${ }^{1} \mathrm{H}$ NMR spectra were acquired at 300,400 , or $500 \mathrm{MHz}$ on a Varian Gemini spectrometer. Chemical shifts are reported in delta $(\delta)$ units in parts per million (ppm) relative to the singlet $(7.24 \mathrm{ppm})$ for chloroform- $d$ and the singlet $(2.04 \mathrm{ppm})$ for acetone$d_{6}$. Splitting patterns are designated as s, singlet; $\mathrm{d}$, doublet; $\mathrm{t}$, triplet; q, quartet; $\mathrm{p}$, quintet; m, multiplet; and br, broad. Coupling constants are recorded in $\mathrm{Hertz}(\mathrm{Hz}) .{ }^{13} \mathrm{C}$ NMR spectra were acquired at 75,100 , or $125 \mathrm{MHz}$ on a Varian Gemini spectrometer. Chemical shifts are reported in ppm relative to the central line of the triplet at $77.0 \mathrm{ppm}$ for chloroform- $d$.

Chiral HPLC analyses were performed on a Thermo Separation Products Spectraseries P100 amd UV100 or P200 and UV200 using Chiralce ${ }^{\boxplus}$ columns (AD, AS, OB-H, OC, OD, or OJ) with heptane / 2-propanol mixtures with ratio of the eluent, flow rate, and column indicated. Retention times $\left(\tau_{R}\right)$ are reported in minutes (min). Chiral GC 
was performed on a Hewlett-Packard 6890 capillary gas chromatograph using a 30m x $0.252 \mathrm{~mm}$ J\&W CyclosilB column. Optical rotations were determined using a JASCO DIP-1000 digital polarimeter in 50-mm cells and the sodium D line $(589 \mathrm{~nm})$ at room temperature in the solvent and concentration indicated.

\section{PART I. Data for cyclization DYKAT methods development:}

Synthesis of 14 and 16:

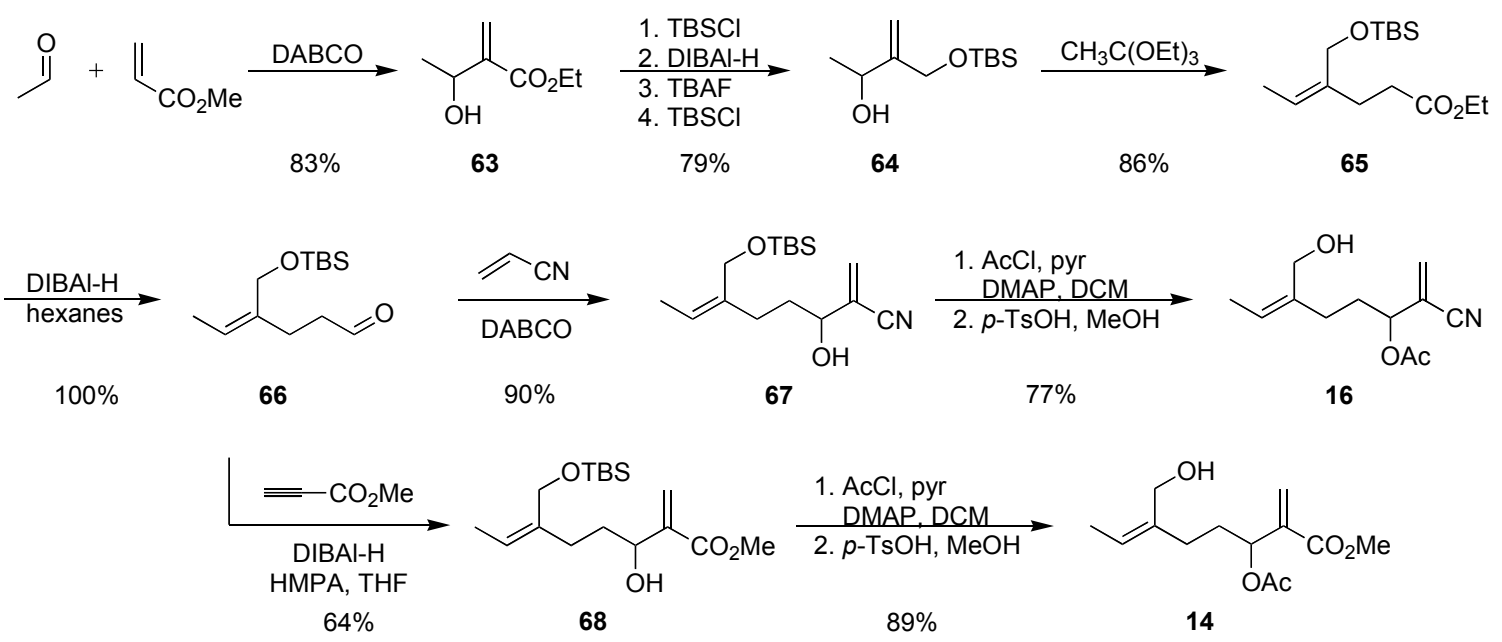

$\overbrace{\text { CoEt }}^{\text {COMpund }}$

Compound 63. DABCO (1.65 g, $14.7 \mathrm{mmol})$ was added to a flask containing acetaldehyde $(2.7 \mathrm{~g}, 60.8 \mathrm{mmol})$ and ethyl acrylate $(17.56 \mathrm{~g}, 175.4 \mathrm{mmol})$. The flask was sealed and stirred at room temperature for 1 week. The resulting solution was diluted with ether and washed 3 times with $1 \mathrm{~N} \mathrm{HCl}$ to remove DABCO. The organic layer was dried over $\mathrm{MgSO}_{4}$, filtered, and concentrated in vacuo. The resulting oily residue was purified via flash chromatography (silica, ether/petroleum ether, gradient) to yield $7.29 \mathrm{~g}$ $(83 \%)$ of 63 as a light yellow oil. $\mathrm{R}_{\mathrm{f}}=0.64$ in $60 \%$ ether/petroleum ether. The obtained spectral data $\left[{ }^{1} \mathrm{H}\right]$ matched literature data. ${ }^{5} \mathrm{IR}$ (film from $\mathrm{CDCl}_{3}$ ): 3449, 1715, 1630, 1452, $1400 \mathrm{~cm}^{-1} .{ }^{1} \mathrm{H}$ NMR $\left(500 \mathrm{MHz}, \mathrm{CDCl}_{3}\right) \delta 6.24(\mathrm{~s}, 1 \mathrm{H}), 5.83(\mathrm{~s}, 1 \mathrm{H}), 4.64(\mathrm{q}, \mathrm{J}=$ $6.5 \mathrm{~Hz}, 1 \mathrm{H}), 4.27(\mathrm{q}, \mathrm{J}=6.5 \mathrm{~Hz}, 2 \mathrm{H}), 1.41(\mathrm{~d}, \mathrm{~J}=6.5 \mathrm{~Hz}, 3 \mathrm{H}), 1.35$ (t, J = 7.0 Hz, 3H). The hydroxyl proton was too broad to be observed.<smiles>C=C(OCC)C(=O)C(C)O[131I]</smiles>

Compound 69. TBSCl $(3.8 \mathrm{~g}, 25 \mathrm{mmol})$ was added to a cooled $\left(0^{\circ} \mathrm{C}\right)$ solution of 63 $(1.73 \mathrm{~g}, 12.0 \mathrm{mmol})$ and imidazole $(1.98 \mathrm{~g}, 29 \mathrm{mmol})$ in DCM $(60 \mathrm{~mL})$. The reaction was removed from the ice bath and stirred at room temperature for $5 \mathrm{~h}$ at which time it was 
quench with saturated aq. ammonium chloride. The resulting biphasic solution was poured into DCM and the aqueous layer was extracted 3 times with DCM. The combined organic layers were dried over magnesium sulfate, filtered, and concentrated in vacuo. The resulting oily residue was purified via flash chromatography (silica, ether/petroleum ether, gradient) to yield $3.04 \mathrm{~g}(98 \%)$ of $\mathbf{6 9}$ as a colorless oil. $\mathrm{R}_{\mathrm{f}}=0.74$ in $20 \%$ ether/petroleum ether. IR (film from $\mathrm{CDCl}_{3}$ ): $1717,1376 \mathrm{~cm}^{-1} .{ }^{1} \mathrm{H}$ NMR $(300 \mathrm{MHz}$, $\left.\mathrm{CDCl}_{3}\right) \delta 6.03(\mathrm{~s}, 1 \mathrm{H}), 5.78(\mathrm{~s}, 1 \mathrm{H}), 4.63(\mathrm{q}, \mathrm{J}=6.3 \mathrm{~Hz}, 1 \mathrm{H}), 4.08(\mathrm{~m}, 2 \mathrm{H}), 1.15(\mathrm{~m}, 6 \mathrm{H})$, 0.74 (s, 9H), -0.09 (s, 3H), -0.12 (s, 3H). ${ }^{13} \mathrm{C}$ NMR $\left(75 \mathrm{MHz}, \mathrm{CDCl}_{3}\right) \delta$ 166.3, 145.5, 123.2, 66.8, 60.5, 25.8 (3C), 24.6, 18.2, 14.2, -4.8 (2C). Anal calcd for $\mathrm{C}_{13} \mathrm{H}_{26} \mathrm{O}_{3} \mathrm{Si}$ : C, $60.42 \%$; H, 10.14\%. Found: C, 60.54\%; H, 10.07\%.<smiles>C=C(CO)C(C)O</smiles>

Compound 70. DIBAl-H ( $160 \mathrm{~mL}$ of $1.0 \mathrm{M}$ in hexanes) was added to a solution cooled $\left(-78^{\circ} \mathrm{C}\right)$ solution of $69(16.3 \mathrm{~g}, 63 \mathrm{mmol})$ in DCM $(240 \mathrm{~mL})$. The reaction was allowed to warm slowly to $0^{\circ} \mathrm{C}$ over $7 \mathrm{~h}$. $\mathrm{MeOH}(120 \mathrm{~mL})$ was added followed by $1000 \mathrm{~mL}$ saturated aq. Rochelle's salt. The resulting cloudy solution was vigorously stirred for $20 \mathrm{~h}$ at which point the emulsions had broken. The resulting aqueous layer was extracted once with DCM and 3 times with ethyl acetate. The combined organic layers were dried over magnesium sulfate, filtered, and concentrated in vacuo to yield $13.4 \mathrm{~g} \mathrm{(98 \% )}$ of primary alcohol as a colorless oil. The crude material was used in the next step without purification. TBAF $\left(90 \mathrm{~mL}\right.$ of $1.0 \mathrm{M}$ in THF) was added to a cooled $\left(0^{\circ} \mathrm{C}\right)$ solution of the crude TBS alcohol $(13.4 \mathrm{~g}, 62 \mathrm{mmol})$ in $120 \mathrm{~mL}$ THF. The resulting solution was removed from the ice bath and stirred for $1.5 \mathrm{~h}$ at which time the reaction was directly concentrated in vacuo and purified via flash chromatography (silica, ethyl acetate/petroleum ether, gradient) to yield $5.99 \mathrm{~g}(95 \%)$ of $\mathbf{7 0}$ as a colorless oil. $\mathrm{R}_{\mathrm{f}}=0.2$ in $100 \%$ ethyl acetate. IR (film from $\mathrm{CDCl}_{3}$ ): $3332,1455,1410 \mathrm{~cm}^{-1}$. ${ }^{1} \mathrm{H}$ NMR (300 $\left.\mathrm{MHz}, \mathrm{CDCl}_{3}\right) \delta 4.96(\mathrm{~s}, 1 \mathrm{H}), 4.94(\mathrm{~s}, 1 \mathrm{H}), 4.30(\mathrm{q}, \mathrm{J}=6.6 \mathrm{~Hz}, 1 \mathrm{H}), 4.16(\mathrm{~d}, \mathrm{~J}=13.2 \mathrm{~Hz}$, $1 \mathrm{H}), 4.04(\mathrm{~d}, \mathrm{~J}=13.2 \mathrm{~Hz}, 1 \mathrm{H}), 2.06($ broad s, $2 \mathrm{H}), 1.21(\mathrm{~d}, \mathrm{~J}=6.3 \mathrm{~Hz}, 3 \mathrm{H}) \cdot{ }^{13} \mathrm{C}$ NMR $\left(75 \mathrm{MHz}, \mathrm{CDCl}_{3}\right) \delta 150.8,111.3,69.7,63.7,22.0$. Anal calcd for $\mathrm{C}_{5} \mathrm{H}_{10} \mathrm{O}_{2}: \mathrm{C}, 58.80 \% ; \mathrm{H}$, 9.87\%. Found: C, 58.68\%; H, 9.98\%.

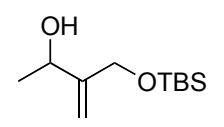

Compound 64. $\mathrm{TBSCl}(8.9 \mathrm{~g}, 59.1 \mathrm{mmol})$ was added to a cooled $\left(0^{\circ} \mathrm{C}\right)$ solution of 70 $(5.99 \mathrm{~g}, 59.1 \mathrm{mmol})$ and imidazole $(4.82 \mathrm{~g}, 70.9 \mathrm{mmol})$ in DCM $(285 \mathrm{~mL})$. The resulting solution was stirred $20 \mathrm{~min}$ at $0^{\circ} \mathrm{C}$ at which point it was quenched with saturated aq. ammonium chloride. The aqueous layer was extracted 3 times with DCM. The combined organic layers were dried over magnesium sulfate, filtered, and concentrated in vacuo. The remaining oily residue was purified via flash chromatography (silica, ether/petroluem ether, gradient) to yield $11.0 \mathrm{~g}(87 \%) 64$ as a colorless oil. $R_{\mathrm{f}}=0.38$ in $30 \%$ ether/petroleum ether. IR (film from $\mathrm{CDCl}_{3}$ ): $3361,1472,1464,837,776 \mathrm{~cm}^{-1} .{ }^{1} \mathrm{H}$ NMR (300 MHz, CDCl $)$ d $4.92(\mathrm{~s}, 1 \mathrm{H}), 4.90(\mathrm{~s}, 1 \mathrm{H}), 4.22(\mathrm{~m}, 1 \mathrm{H}), 4.16(\mathrm{~d}, \mathrm{~J}=12.9 \mathrm{~Hz}$, 
1H), $4.08(\mathrm{~d}, \mathrm{~J}=13.2 \mathrm{~Hz}, 1 \mathrm{H}), 2.36(\mathrm{~d}, \mathrm{~J}=4.8 \mathrm{~Hz}, 1 \mathrm{H}), 1.19(\mathrm{~d}, \mathrm{~J}=6.6 \mathrm{~Hz}, 3 \mathrm{H}), 0.76(\mathrm{~s}$, 9H), $0.06(\mathrm{~s}, 6 \mathrm{H}) .{ }^{13} \mathrm{C} \mathrm{NMR}\left(75 \mathrm{MHz}, \mathrm{CDCl}_{3}\right) \delta 150.6,110.1,69.6,64.9,23.8(3 \mathrm{C}), 21.9$, $18.2,-5.5$ (2C). Anal calcd for $\mathrm{C}_{11} \mathrm{H}_{24} \mathrm{O}_{2} \mathrm{Si}$ : C, 61.05\%; H, 11.18\%. Found: $\mathrm{C}, 61.22 \%$; $\mathrm{H}$, $11.32 \%$.

$\mathrm{CO}_{2} \mathrm{Et}$

Compound 65. Triethyl orthoacetate $(120 \mathrm{~mL})$ was added to a flask containing 64 (10.6 $\mathrm{g}, 48.9 \mathrm{mmol})$. The flask was capped with a cold water condenser and placed into an oil bath at $140^{\circ} \mathrm{C}$. The reaction was stirred for $10 \mathrm{~h}$. The excess triethylorthoformate was removed by distillation and the resulting oily residue was passed through a short plug of silica, eluting with $10 \%$ ether/petroleum ether to yield $11.71 \mathrm{~g}(86 \%)$ of 65 as a colorless oil. $\mathrm{R}_{\mathrm{f}}=0.50$ in $8 \%$ ether/petroleum ether, $\mathrm{KMnO}_{4}$ stain. The obtained spectral data [IR, ${ }^{1} \mathrm{H}$ ] matched literature values. ${ }^{6}$ IR (film): 1739, 1472, $837 \mathrm{~cm}^{-1}{ }^{1} \mathrm{H}$ NMR $(300 \mathrm{MHz}$, $\left.\mathrm{CDCl}_{3}\right) \delta 5.15(\mathrm{q}, \mathrm{J}=6.9 \mathrm{~Hz}, 1 \mathrm{H}), 4.03(\mathrm{~s}, 2 \mathrm{H}), 3.95(\mathrm{q}, \mathrm{J}=7.2 \mathrm{~Hz}, 2 \mathrm{H}), 2.26(\mathrm{~s}, 4 \mathrm{H})$, $1.44(\mathrm{~d}, \mathrm{~J}=6.9 \mathrm{~Hz}, 3 \mathrm{H}), 1.08(\mathrm{t}, \mathrm{J}=7.2 \mathrm{~Hz}, 3 \mathrm{H}), 0.74(\mathrm{~s}, 9 \mathrm{H}),-0.09(\mathrm{~s}, 6 \mathrm{H}) .{ }^{13} \mathrm{C} \mathrm{NMR}$ $\left(75 \mathrm{MHz}, \mathrm{CDCl}_{3}\right) \delta 173.6,137.7,121.0,60.1,60.0,33.4,29.9,25.9$ (3C), 18.3, 14.2, 13.0, -4.8 (2C). Anal calcd for $\mathrm{C}_{15} \mathrm{H}_{30} \mathrm{O}_{3} \mathrm{Si}: \mathrm{C}, 62.89 \%$; H, 10.55\%. Found: $\mathrm{C}, 62.86 \%$; $\mathrm{H}$, $10.60 \%$.

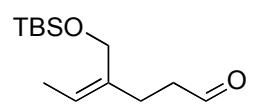

Compound 66. DIBAl-H ( $43 \mathrm{~mL}$ of $1.0 \mathrm{M}$ in hexanes) was added dropwise via syringe pump over $45 \mathrm{~min}$ to cooled $\left(-78^{\circ} \mathrm{C}\right)$ solution of $\mathbf{6 5}(11.7 \mathrm{~g}, 40.9 \mathrm{mmol})$ in $200 \mathrm{~mL}$ of hexanes. The resulting colorless solution was stirred at $-78^{\circ} \mathrm{C}$ for $1.5 \mathrm{~h}$ (until TLC indicated complete consumption of ester) at which time the reaction was quenched by the dropwise addition of cooled $\left(-78^{\circ} \mathrm{C}\right) \mathrm{MeOH}(30 \mathrm{~mL})$ via syringe pump over $30 \mathrm{~min}$. The resulting cloudy solution was stirred $20 \mathrm{~min}$ at $-78^{\circ} \mathrm{C}$ at which time $24 \mathrm{~mL}$ of $4 \mathrm{M}$ aq. $\mathrm{NaH}_{2} \mathrm{PO}_{4}$ was added. The flask was removed from the dry ice bath and allowed to warm to room temperature while vigorously stirring. The reaction was diluted with $150 \mathrm{~mL}$ of ethyl acetate and stirred at room temperature for $5 \mathrm{~h}$ (until the gummy solid became a white flaky solid). The heterogeneous solution was filtered through a short plug of celite eluting with ethyl acetate. The filtrate was dried over $\mathrm{MgSO}_{4}$, filtered, and concentrated in vacuo to yield $9.9 \mathrm{~g}$ (quantitative yield) of analytically pure 66 as a light yellow oil.

IR (film from $\left.\mathrm{CDCl}_{3}\right): 2712,1728,1472,939,837 \mathrm{~cm}^{-1} .{ }^{1} \mathrm{H} \mathrm{NMR}\left(300 \mathrm{MHz}, \mathrm{CDCl}_{3}\right) \delta$ $9.60(\mathrm{~s}, 1 \mathrm{H}), 5.16(\mathrm{q}, \mathrm{J}=6.9 \mathrm{~Hz}, 1 \mathrm{H}), 4.04(\mathrm{~s}, 2 \mathrm{H}), 2.41(\mathrm{t}, \mathrm{J}=7.5 \mathrm{~Hz}, 2 \mathrm{H}), 2.83(\mathrm{t}, \mathrm{J}=$ $6.9 \mathrm{~Hz}, 2 \mathrm{H}), 1.46(\mathrm{~d}, \mathrm{~J}=6.9 \mathrm{~Hz}, 3 \mathrm{H}), 0.74(\mathrm{~s}, 9 \mathrm{H}), 0.09(\mathrm{~s}, 6 \mathrm{H}) .{ }^{13} \mathrm{C} \mathrm{NMR}(75 \mathrm{MHz}$, $\left.\mathrm{CDCl}_{3}\right) \delta 202.9,137.4,121.3,60.2,42.5,27.2,25.9$ (3C), 18.3, 13.0, -5.4 (2C). Anal calcd for $\mathrm{C}_{13} \mathrm{H}_{26} \mathrm{O}_{2} \mathrm{Si}$ : C, 64.41\%; H, 10.81\%. Found: C, 64.34\%; H, 10.80\%. 
<smiles>C=C(C#N)C(O)CC/C(=C/C)COC(C)(C)C</smiles>

Compound 67. DABCO $(0.69 \mathrm{~g}, 5.5 \mathrm{mmol})$ was added to a pressure flask containing 66 $(5.0 \mathrm{~g}, 20.6 \mathrm{mmol})$ and acrylonitrile $(21 \mathrm{~mL})$. The flask was sealed and heated at $45^{\circ} \mathrm{C}$ for 1 week. The resulting solution was diluted with ether and washed 2 times with $1 \mathrm{~N} \mathrm{HCl}$ to remove the DABCO. The organic layer was dried over magnesium sulfate and the volatile organic compounds were removed via distillation. The resulting crude material was purified via flash chromatography (silica, $10-50 \%$ ether/petroleum ether, gradient) to yield $3.78 \mathrm{~g}(49 \%)$ of $\mathbf{6 7}$ as a colorless oil. Note: when the reaction was performed with $0.19 \mathrm{~g}$ DABCO, $1.14 \mathrm{~g} \mathrm{66}$, and $4.7 \mathrm{~mL}$ acrylonitrile, $1.24 \mathrm{~g}(90 \%)$ of 67 was isolated. $\mathrm{R}_{\mathrm{f}}$ $=0.51$ in $50 \%$ ether/petroleum ether. IR (film from $\mathrm{CH}_{2} \mathrm{Cl}_{2}$ ): $3444,2227,1742,837,776$ $\mathrm{cm}^{-1} .{ }^{1} \mathrm{H}$ NMR $\left(300 \mathrm{MHz}, \mathrm{CDCl}_{3}\right) \delta 5.94(\mathrm{~s}, 1 \mathrm{H}), 5.87(\mathrm{~s}, 1 \mathrm{H}), 5.34(\mathrm{q}, \mathrm{J}=6.9 \mathrm{~Hz}, 1 \mathrm{H})$, $4.16(\mathrm{~m}, 2 \mathrm{H}), 4.04(\mathrm{~m}, 1 \mathrm{H}), 2.16(\mathrm{t}, \mathrm{J}=6.9 \mathrm{~Hz}, 2 \mathrm{H}), 1.86(\mathrm{~m}, 1 \mathrm{H}), 1.69(\mathrm{~m}, 1 \mathrm{H}), 1.55(\mathrm{~d}$, $\mathrm{J}=6.9 \mathrm{~Hz}, 3 \mathrm{H}), 0.83(\mathrm{~s}, 9 \mathrm{H}), 0.02(\mathrm{~s}, 6 \mathrm{H})$. The hydroxyl proton was too broad to be observed. ${ }^{13} \mathrm{C}$ NMR $\left(75 \mathrm{MHz}, \mathrm{CDCl}_{3}\right) \delta$ 137.1, 129.4, 126.8, 123.3, 117.4, 71.6, 60.7, 34.3, 31.1, 25.9 (3C), 18.4, 13.2, -5.4 (2C). Anal calcd for $\mathrm{C}_{16} \mathrm{H}_{29} \mathrm{NO}_{2} \mathrm{Si}: \mathrm{C}, 65.03 \%$; $\mathrm{H}$, 9.89\%; N, 4.74\%. Found: C, 65.15\%; H, 10.02\%; N, 4.85\%.<smiles>C=C(C#N)C(CC/C(=C/C)COC(C)(C)C)OC</smiles>

Compound 71. Acetyl chloride $(1.47 \mathrm{~mL}, 20.7 \mathrm{mmol})$ was added dropwise to a cooled $\left(0^{\circ} \mathrm{C}\right)$ solution of $67(3.30 \mathrm{~g}, 11.17 \mathrm{mmol})$, pyridine $(2.4 \mathrm{~mL}, 33.6 \mathrm{mmol})$, and DMAP $(0.54 \mathrm{~g}, 4.5 \mathrm{mmol})$ in $60 \mathrm{~mL}$ DCM. The resulting solution was stirred at $0^{\circ} \mathrm{C}$ for $1.5 \mathrm{~h}$ at which time it was quenched with saturated aq. ammonium chloride and poured into methylene chloride. The aqueous layer was extracted 3 times with DCM. The combined organic layers were dried over $\mathrm{MgSO}_{4}$, filtered, and concentrated in vacuo. The resulting oily residue was purified via flash chromatography (silica, ether/petroleum ether, gradient) to yield $3.38 \mathrm{~g}(90 \%)$ of 71 as a colorless oil. $\mathrm{R}_{\mathrm{f}}=0.80$ in $25 \%$ ether/petroleum ether. IR (film from $\mathrm{CDCl}_{3}$ ): 1750, 837, $776 \mathrm{~cm}^{-1} .{ }^{1} \mathrm{H}$ NMR $\left(300 \mathrm{MHz}, \mathrm{CDCl}_{3}\right) \delta 5.94(\mathrm{~s}$, $1 \mathrm{H}), 5.88(\mathrm{~s}, 1 \mathrm{H}), 5.18(\mathrm{~m}, 2 \mathrm{H}), 4.09(\mathrm{~s}, 2 \mathrm{H}), 2.04(\mathrm{~m}, 2 \mathrm{H}), 2.01(\mathrm{~s}, 3 \mathrm{H}), 1.82(\mathrm{~m}, 2 \mathrm{H})$, $1.53(\mathrm{~d}, \mathrm{~J}=6.9 \mathrm{~Hz}, 3 \mathrm{H}), 0.81(\mathrm{~s}, 9 \mathrm{H}), 0.02(\mathrm{~s}, 6 \mathrm{H}) .{ }^{13} \mathrm{CNMR}\left(100 \mathrm{MHz}, \mathrm{CDCl}_{3}\right) \delta 169.9$, $137.3,132.6,122.9,121.6,116.2,73.1,60.0,31.4,30.0,25.9$ (3C), 20.9, 18.3, 13.1, -5.4 (2C). This compound was fully characterized after conversion to $\mathbf{1 6}$.<smiles>C=C(C#N)C(CC/C(=C/C)CO)O[Na]</smiles>

Compound 16. $p$-Toluenesulfonic acid $(0.51 \mathrm{~g}, 2.7 \mathrm{mmol})$ was added to a solution of 71 (3.38 g, $10.0 \mathrm{mmol})$ in $60 \mathrm{~mL} \mathrm{MeOH}$. The resulting solution was stirred $1 \mathrm{~h}$ at room 
temperature at which time it was directly concentrated in vacuo. The resulting oily residue was purified via flash chromatography (silica, ether/petroleum ether, gradient) to yield $1.92 \mathrm{~g}(86 \%)$ of $\mathbf{1 6}$ as a colorless oil. When the reaction was performed with 0.017 g $p$-toluenesulfonic acid, $0.116 \mathrm{~g} \mathrm{71}$, and $2.4 \mathrm{~mL} \mathrm{MeOH}, 0.0745 \mathrm{~g} \mathrm{(99 \% )} \mathrm{of} 16$ was obtained. From the kinetic resolution, recovered 16 was of $99 \%$ ee, $[\alpha]_{D}-23.70(c=1.08$, $\mathrm{CH}_{2} \mathrm{Cl}_{2}$ ). The enantiomeric excess was determined via HPLC analysis of a derivative (72). $\mathrm{R}_{\mathrm{f}}=0.1$ in $50 \%$ ether/petroleum ether. IR (film from $\mathrm{CDCl}_{3}$ ): $3445,2228,1748$, $977 \mathrm{~cm}^{-1} .{ }^{1} \mathrm{H}$ NMR $\left(300 \mathrm{MHz}, \mathrm{CDCl}_{3}\right) \delta 5.96(\mathrm{~s}, 1 \mathrm{H}), 5.90(\mathrm{~s}, 1 \mathrm{H}), 5.32(\mathrm{q}, \mathrm{J}=6.9 \mathrm{~Hz}$, $1 \mathrm{H}), 5.19(\mathrm{t}, \mathrm{J}=6.9 \mathrm{~Hz}, 1 \mathrm{H}), 4.09(\mathrm{~d}, \mathrm{~J}=3.3 \mathrm{~Hz}, 2 \mathrm{H}), 2.08(\mathrm{~m}, 2 \mathrm{H}), 2.02(\mathrm{~s}, 3 \mathrm{H}), 1.85$ $(\mathrm{m}, 2 \mathrm{H}), 1.59(\mathrm{~d}, \mathrm{~J}=6.9 \mathrm{~Hz}, 3 \mathrm{H})$. The hydroxyl proton was too broad to be observed. ${ }^{13} \mathrm{C}$ NMR $\left(75 \mathrm{MHz}, \mathrm{CDCl}_{3}\right) \delta 169.9,136.9,132.9,123.9,122.6,116.1,72.9,59.6,31.3,30.4$, 20.9, 13.1. Anal calcd for $\mathrm{C}_{12} \mathrm{H}_{17} \mathrm{NO}_{3}$ : C, 64.55\%; H, 7.67. Found: C, 64.38\%; H, 7.57\%.

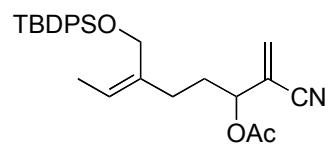

Compound 72. TBDPSCl $(12 \mu \mathrm{L}, 0.046 \mathrm{mmol})$ was added to a cooled $\left(0^{\circ} \mathrm{C}\right)$ solution of $16(0.010 \mathrm{~g}, 0.045 \mathrm{mmol})$ and imidazole $(0.004 \mathrm{~g}, 0.059 \mathrm{mmol})$ in $0.4 \mathrm{~mL}$ DCM. The resulting solution was stirred $3 \mathrm{~h}$ at room temperature at which time it was directly purified via flash chromatography to yield $0.018 \mathrm{~g},(90 \%)$ of 72 as a colorless oil. The enantiomeric excess was determined to be $99 \%$ ee, $[\alpha]_{\mathrm{D}}-12.44\left(\mathrm{c}=1.50, \mathrm{CH}_{2} \mathrm{Cl}_{2}\right)$, by HPLC. (Chiralcel AD column, 99:1 heptane:iPrOH, flow rate $=1.0 \mathrm{~mL} / \mathrm{min}, 254 \mathrm{~nm}, \mathrm{t}_{\mathrm{r}}$ : 5.35 (minor), 5.83 (major)). $R_{\mathrm{f}}=0.8$ in $50 \%$ ether/petroleum ether. IR (neat): 1749,1472 , $741 \mathrm{~cm}^{-1} .{ }^{1} \mathrm{H}$ NMR $\left(500 \mathrm{MHz}, \mathrm{CDCl}_{3}\right) \delta 7.65(\mathrm{~m}, 4 \mathrm{H}), 7.39(\mathrm{~m}, 6 \mathrm{H}), 6.00(\mathrm{~s}, 1 \mathrm{H}), 5.93$ $(\mathrm{s}, 1 \mathrm{H}), 5.25(\mathrm{~m}, 2 \mathrm{H}), 4.12(\mathrm{~s}, 2 \mathrm{H}), 2.21(\mathrm{~m}, 2 \mathrm{H}), 2.07(\mathrm{~s}, 3 \mathrm{H}), 1.99(\mathrm{~m}, 1 \mathrm{H}), 1.90(\mathrm{~m}$, $1 \mathrm{H}), 1.39(\mathrm{~d}, \mathrm{~J}=6.5 \mathrm{~Hz}, 3 \mathrm{H}), 1.02(\mathrm{~s}, 9 \mathrm{H}) .{ }^{13} \mathrm{C} \mathrm{NMR}\left(125 \mathrm{MHz}, \mathrm{CDCl}_{3}\right) \delta 169.9,136.9$, $135.6,134.8,133.5,132.6,129.7,127.7,122.9,121.9,116.2,73.1,60.8,31.4,30.0,26.8$, 20.9, 19.2, 13.0 .

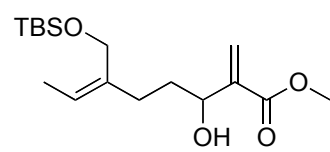

Compound 68. Baylis Hillman: DABCO (0.042 g, $0.37 \mathrm{mmol})$ was added to a pressure tube containing $66(0.30 \mathrm{~g}, 1.23 \mathrm{mmol})$ and methyl acrylate $(1.1 \mathrm{ml})$. The tube was sealed and heated at $50^{\circ} \mathrm{C}$ for two weeks. The resulting solution was directly applied to flash chromatography (silica, ether/petroleum ether, gradient) to yield $0.030 \mathrm{~g}$ Baylis Hillman adduct, $8 \%$ (45\% based on recovered 66$)$ of $\mathbf{6 8}$ as a colorless oil.

Hydroalumination: DIBAl-H (77.4 $\mathrm{mL}$ of $1.0 \mathrm{M}$ in hexanes) was added dropwise to a cooled $\left(0^{\circ} \mathrm{C}\right)$ solution of HMPA $(42 \mathrm{~mL}, 240 \mathrm{mmol})$ in $250 \mathrm{~mL}$ THF. The resulting solution was stirred $1 \mathrm{~h}$ at $0^{\circ} \mathrm{C}$. Freshly distilled methyl propiolate $(7.32 \mathrm{~mL}, 88 \mathrm{mmol})$ was added at which point a light yellow color appeared. The resulting solution was stirred 
an additional $1.5 \mathrm{~h}$ at $0^{\circ} \mathrm{C}$ at which time a solution of $66(6.25 \mathrm{~g}, 25.8 \mathrm{mmol})$ in THF (25 $\mathrm{mL}$ ) was added dropwise. After the addition was complete, the flask was removed from the ice bath and stirred at room temperature for $5 \mathrm{~h}$. The reaction was then cooled to $0^{\circ} \mathrm{C}$ and quenched via dropwise addition of $1 \mathrm{~N} \mathrm{HCl}$. The resulting aqueous layer was extracted 3 times with ether. The combined organic layers were washed with saturated aq. $\mathrm{NaHCO}_{3}$, dried over $\mathrm{MgSO}_{4}$, filtered, and concentrated in vacuo. The resulting oily residue was purified via flash chromatography to yield $5.40 \mathrm{~g},(64 \%) \mathbf{6 8}$ as a colorless oil. $\mathrm{R}_{\mathrm{f}}=0.4$ in $30 \%$ ether/petroleum ether. IR (neat): $3456,1721,1439,837,775 \mathrm{~cm}^{-1} .{ }^{1} \mathrm{H}$ NMR (300 MHz, $\left.\mathrm{CDCl}_{3}\right) \delta 6.13(\mathrm{~s}, 1 \mathrm{H}), 5.74(\mathrm{~s}, 1 \mathrm{H}), 5.27(\mathrm{q}, \mathrm{J}=6.9 \mathrm{~Hz}, 1 \mathrm{H}), 4.31(\mathrm{~m}$, $1 \mathrm{H}), 4.10$ (q, J = $11.7 \mathrm{~Hz}, 2 \mathrm{H}), 3.67$ (s, 3H), $2.10(\mathrm{~m}, 2 \mathrm{H}), 1.75(\mathrm{~m}, 1 \mathrm{H}), 1.63(\mathrm{~m}, 1 \mathrm{H})$, $1.06(\mathrm{~d}, \mathrm{~J}=6.9 \mathrm{~Hz}, 3 \mathrm{H}), 0.81(\mathrm{~s}, 9 \mathrm{H}),-0.03(\mathrm{~s}, 6 \mathrm{H})$. The hydroxyl proton was too broad to be observed. ${ }^{13} \mathrm{C} \mathrm{NMR}\left(75 \mathrm{MHz}, \mathrm{CDCl}_{3}\right) \delta 167.0,142.5,138.3,124.9,121.6,71.2$, $60.3,51.8,34.8,31.1,25.9(3 \mathrm{C}), 18.4,13.1,-5.4(2 \mathrm{C})$. Anal calcd for $\mathrm{C}_{17} \mathrm{H}_{32} \mathrm{O}_{4} \mathrm{Si}$ : C, $62.15 \%$; H, 9.82\%. Found: C, 62.29\%; H, 9.62\%.

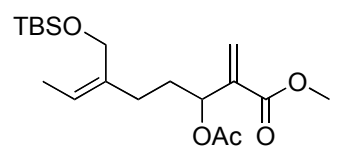

Compound 73. Acetyl chloride $(2.1 \mathrm{~mL}, 29 \mathrm{mmol})$ was added dropwise to a cooled $\left(0^{\circ} \mathrm{C}\right)$ solution of $68(5.1 \mathrm{~g}, 15.5 \mathrm{mmol})$, pyridine $(2.8 \mathrm{~mL}, 39 \mathrm{mmol})$, and DMAP $(0.76 \mathrm{~g}$, $6.2 \mathrm{mmol})$ in $80 \mathrm{~mL}$ DCM. The resulting solution was stirred at $0^{\circ} \mathrm{C}$ for $1 \mathrm{~h}$ at which time it was quenched with saturated aq. ammonium chloride and poured into methylene chloride. The aqueous layer was extracted 3 times with DCM. The combined organic layers were dried over $\mathrm{MgSO}_{4}$, filtered, and concentrated in vacuo. The resulting oily residue was purified via flash chromatography (silica, $0-35 \%$ ether/petroleum ether, gradient) to yield $5.22 \mathrm{~g}(91 \%)$ of 73 as a colorless oil. $\mathrm{R}_{\mathrm{f}}=0.70 \mathrm{in} 30 \%$ ether/petroleum ether. IR (film from $\left.\mathrm{CDCl}_{3}\right): 1749,1722,837,776 \mathrm{~cm}^{-1} .{ }^{1} \mathrm{H}$ NMR $\left(300 \mathrm{MHz}, \mathrm{CDCl}_{3}\right) \delta$ $6.18(\mathrm{~s}, 1 \mathrm{H}), 5.66(\mathrm{~s}, 1 \mathrm{H}), 5.49(\mathrm{dd}, \mathrm{J}=3.9,8.1 \mathrm{~Hz}, 1 \mathrm{H}), 5.19(\mathrm{q}, \mathrm{J}=6.9 \mathrm{~Hz}, 1 \mathrm{H}), 4.09$ (s, 2H), $3.67(\mathrm{~s}, 3 \mathrm{H}), 2.06(\mathrm{~m}, 2 \mathrm{H}), 1.98(\mathrm{~s}, 3 \mathrm{H}), 1.75(\mathrm{~m}, 2 \mathrm{H}), 1.51(\mathrm{~d}, \mathrm{~J}=7.5 \mathrm{~Hz}, 3 \mathrm{H}), 0.80$ $(\mathrm{s}, 9 \mathrm{H}),-0.03(\mathrm{~s}, 6 \mathrm{H}) .{ }^{13} \mathrm{C} \mathrm{NMR}\left(75 \mathrm{MHz} \mathrm{CDCl}_{3}\right) \delta 169.9,165.7,140.2,138.1,125.1$, $120.8,71.6,59.9,51.9,32.8,30.2,25.9$ (3C), 21.1, 18.3, 13.1, -5.4 (2C). This compound was fully characterized after conversion to $\mathbf{1 4}$.

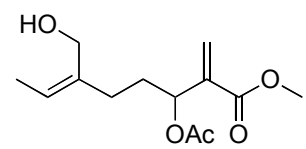

Compound 14. $p$-Toluenesulfonic acid ( $0.66 \mathrm{~g}, 3.5 \mathrm{mmol})$ was added to a solution of 73 (5.22 g, $14.1 \mathrm{mmol})$ in $100 \mathrm{~mL} \mathrm{MeOH}$. The resulting solution was stirred $1 \mathrm{~h}$ at room temperature at which time it was directly concentrated in vacuo. The resulting oily residue was purified via flash chromatography (silica, 10\%-70\% ether/petroleum ether, gradient) to yield $3.18 \mathrm{~g}(88 \%)$ of $\mathbf{1 4}$ as a colorless oil. Note: when the reaction was 
performed with $0.0017 \mathrm{~g} p$-toluenesulfonic acid, $0.015 \mathrm{~g} \mathrm{73}$, and $0.25 \mathrm{~mL} \mathrm{MeOH}, 0.010 \mathrm{~g}$ $(98 \%)$ of 14 was isolated. From the kinetic resolution, recovered 14 was of $91 \%$ ee, $[\alpha]_{D}$ $+1.53\left(\mathrm{c}=2.11, \mathrm{CH}_{2} \mathrm{Cl}_{2}\right)$. The enantiomeric excess was determined via HPLC analysis. (Chiralcel OD column, 97:3 heptane:iPrOH, flow rate $=1.0 \mathrm{~mL} / \mathrm{min}, 220 \mathrm{~nm}, \mathrm{t}_{\mathrm{r}}: 23.55$ (major), 26.60 (minor)). $\mathrm{R}_{\mathrm{f}}=0.08$ in 50\% ether/petroleum ether. IR (neat): 3422,1721 , 1439, $976 \mathrm{~cm}^{-1} .{ }^{1} \mathrm{H}$ NMR $\left(300 \mathrm{MHz}, \mathrm{CDCl}_{3}\right) \delta 6.19(\mathrm{~s}, 1 \mathrm{H}), 5.68(\mathrm{~s}, 1 \mathrm{H}), 5.49(\mathrm{dd}, \mathrm{J}=$ $3.9,8.1 \mathrm{~Hz}, 1 \mathrm{H}), 5.31(\mathrm{q}, \mathrm{J}=6.9 \mathrm{~Hz}, 1 \mathrm{H}), 4.07(\mathrm{~s} 2 \mathrm{H}), 3.68(\mathrm{~s}, 3 \mathrm{H}), 2.08(\mathrm{~m}, 2 \mathrm{H}), 1.99(\mathrm{~s}$, $3 \mathrm{H}), 1.85(\mathrm{~m}, 1 \mathrm{H}), 1.71(\mathrm{~m}, 1 \mathrm{H}), 1.58(\mathrm{~d}, \mathrm{~J}=6.9 \mathrm{~Hz}, 3 \mathrm{H})$. The hydroxyl proton was too broad to be observed. ${ }^{13} \mathrm{C}$ NMR $\left(75 \mathrm{MHz}, \mathrm{CDCl}_{3}\right) \delta 167.0,165.8,140.0,137.6,125.2$, 123.9, 71.3, 60.0, 52.0, 32.7, 31.3, 21.1, 13.2. HRMS calculated for $\mathrm{C}_{11} \mathrm{H}_{17} \mathrm{O}_{3}\left(\mathrm{M}^{+}-\mathrm{OAc}\right)$ : 197.1178. Found 197.1141. Anal calcd for $\mathrm{C}_{13} \mathrm{H}_{20} \mathrm{O}_{5}: \mathrm{C}, 60.92 \%$; H, 7.87. Found: $\mathrm{C}$, $61.18 \%$; H, 7.82\%.

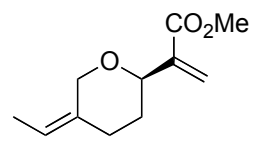

Compound 15. A solution of $(\mathrm{Pd}(\pi-\mathrm{allyl}) \mathrm{Cl})_{2}(0.00084 \mathrm{~g}, 0.0023 \mathrm{mmol})$ and $(R, R)-\mathbf{L}-\mathbf{4}$ $(0.0055 \mathrm{~g}, 0.0071 \mathrm{mmol})$ in $0.5 \mathrm{~mL}$ degassed dioxane was added via cannula to a solution of $14(0.030 \mathrm{~g}, 0.12 \mathrm{mmol})$, and tetrahexylammonium chloride $(0.014 \mathrm{~g}, 0.036 \mathrm{mmol})$ in degassed dioxane $(0.7 \mathrm{~mL})$. The reaction was stirred at $80^{\circ} \mathrm{C}$ under argon for $17 \mathrm{~h}$. The resulting light yellow solution was purified directly via flash chromatography (silica, ether/petroleum ether, gradient) to yield $0.022 \mathrm{~g}(92 \%)$ of 15 as a colorless oil. $[\alpha]_{\mathrm{D}}$ $+69.28\left(\mathrm{c}=0.67, \mathrm{CH}_{2} \mathrm{Cl}_{2}\right)$. The enantiomeric excess was determined to be $95 \%$ via chiral $\mathrm{GC}$ analysis. (Cyclosil B column, $120^{\circ} \mathrm{C}$ isotherm, flow rate $=2.0 \mathrm{~mL} / \mathrm{min}, \mathrm{t}_{\mathrm{r}}: 26.19$ (major), 27.47 (minor)). $\mathrm{R}_{\mathrm{f}}=0.70$ in $25 \%$ ether/petroleum ether. IR (film from $\mathrm{CDCl}_{3}$ ): $1721,1382 \mathrm{~cm}^{-1} .{ }^{1} \mathrm{H}$ NMR $\left(400 \mathrm{MHz}, \mathrm{CDCl}_{3}\right) \delta 6.23(\mathrm{~s}, 1 \mathrm{H}), 5.80(\mathrm{~s}, 1 \mathrm{H}), 5.27$ (app q, J $=6.8 \mathrm{~Hz}, 1 \mathrm{H}), 4.71(\mathrm{~d}, \mathrm{~J}=12.8 \mathrm{~Hz}, 1 \mathrm{H}), 4.31(\mathrm{~d}, \mathrm{~J}=10.8 \mathrm{~Hz}, 1 \mathrm{H}), 3.86(\mathrm{~d}, \mathrm{~J}=12.8 \mathrm{~Hz}$, $1 \mathrm{H}), 3.75(\mathrm{~s}, 3 \mathrm{H}), 2.32(\mathrm{~m}, 2 \mathrm{H}), 2.00(\mathrm{~m}, 1 \mathrm{H}), 1.60(\mathrm{~d}, \mathrm{~J}=8.4 \mathrm{~Hz}, 3 \mathrm{H}), 1.35(\mathrm{~m}, 1 \mathrm{H}) .{ }^{13} \mathrm{C}$ NMR $\left(100 \mathrm{MHz}, \mathrm{CDCl}_{3}\right) \delta 166.4,141.5,133.6,124.6,118.9,75.4,66.7,51.8,33.9,33.1$, 12.6. HRMS calculated for $\mathrm{C}_{11} \mathrm{H}_{16} \mathrm{O}_{3}\left(\mathrm{M}^{+}\right)$: 196.1099 . Found 196.1089.

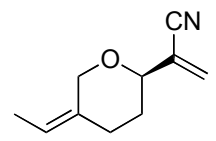

Compound 17. A solution of $(\mathrm{Pd}(\pi-\mathrm{allyl}) \mathrm{Cl})_{2}(0.00084 \mathrm{~g}, 0.0023 \mathrm{mmol})$ and $(R, R)-\mathbf{L}-\mathbf{4}$ $(0.0050 \mathrm{~g}, 0.0071 \mathrm{mmol})$ in $0.5 \mathrm{~mL}$ degassed toluene was added via cannula to a solution of $16(0.027 \mathrm{~g}, 0.12 \mathrm{mmol})$, and tetrahexylammonium chloride $(0.014 \mathrm{~g}, 0.036 \mathrm{mmol})$ in degassed toluene $(0.7 \mathrm{~mL})$. The reaction was stirred at $80^{\circ} \mathrm{C}$ under argon for $17 \mathrm{~h}$. The resulting light yellow solution was purified directly via flash chromatography (silica, ether/petroleum ether, gradient) to yield $0.018 \mathrm{~g}(94 \%)$ of 17 as a colorless oil. $[\alpha]_{\mathrm{D}}$ $+45.74\left(\mathrm{c}=0.82, \mathrm{CH}_{2} \mathrm{Cl}_{2}\right)$. The enantiomeric excess was determined to be $98 \%$ via chiral 
$\mathrm{GC}$ analysis. (Cyclosil B column, $110^{\circ} \mathrm{C}$ isotherm, flow rate $=2.0 \mathrm{~mL} / \mathrm{min}$, $\mathrm{t}_{\mathrm{r}}: 37.97$ (major), 45.06 (minor)). The enantiomeric excess can also be determined via HPLC analysis. (Chiralcel OD column, 99.9:0.1 heptane: $\mathrm{PrOH}$, flow rate $=1.0 \mathrm{~mL} / \mathrm{min}$, $220 \mathrm{~nm}, \mathrm{t}_{\mathrm{r}}: 15.08$ (major), 16.87 (minor)). $\mathrm{R}_{\mathrm{f}}=0.60$ in $25 \%$ ether/petroleum ether. IR (film from $\mathrm{CDCl}_{3}$ ): 2227, 1622, 1442, 1386, $954 \mathrm{~cm}^{-1} .{ }^{1} \mathrm{H}$ NMR (300 MHz, $\left.\mathrm{CDCl}_{3}\right) \delta$ $6.01(\mathrm{~s}, 1 \mathrm{H}), 5.97(\mathrm{~s}, 1 \mathrm{H}), 5.34(\mathrm{~m}, 1 \mathrm{H}), 4.72(\mathrm{~d}, \mathrm{~J}=12.9 \mathrm{~Hz}, 1 \mathrm{H}), 4.03(\mathrm{~d}, \mathrm{~J}=11.1 \mathrm{~Hz}$, $1 \mathrm{H}), 4.85(\mathrm{~d}, \mathrm{~J}=12.6 \mathrm{~Hz}, 1 \mathrm{H}), 2.36(\mathrm{~m}, 2 \mathrm{H}), 2.02(\mathrm{~m}, 1 \mathrm{H}), 1.62(\mathrm{~d}, \mathrm{~J}=6.9 \mathrm{~Hz}, 3 \mathrm{H}), 1.55$ (m, 1H). ${ }^{13} \mathrm{C}$ NMR $\left(75 \mathrm{MHz}, \mathrm{CDCl}_{3}\right) \delta 132.2,129.9,124.5,112.0,117.1,76.4,66.5$, 32.5, 32.3, 12.7. Anal calcd for $\mathrm{C}_{10} \mathrm{H}_{13} \mathrm{NO}$ : C, $73.59 \%$; H, 8.03. Found: $\mathrm{C}, 73.38 \%$; $\mathrm{H}$, $8.00 \%$.

\section{PART II. Data for stereochemistry rationale:}

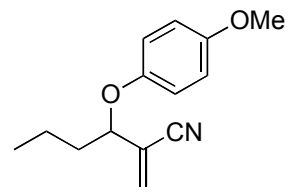

Compound 19: $p$-Methoxyphenol (50 mg, $0.40 \mathrm{mmol}),(S, S)-\mathbf{L}-3$ ( $8.4 \mathrm{mg}, 12 \mu \mathrm{mol})$ and $\mathrm{Pd}_{2} \mathrm{dba}_{3} \cdot \mathrm{CHCl}_{3}(4.1 \mathrm{mg}, 4.0 \mu \mathrm{mol})$ were weighed into a test-tube directly. The test-tube was put under vacuum and refilled with argon (repeated 3 times). Allylic carbonate $\mathbf{1 8}^{7}$ (64 mg, $0.28 \mathrm{mmol}$ ) was weighed into another test-tube and methylene chloride $(2.8 \mathrm{~mL})$ was added. The solution was purged with argon for $15 \mathrm{~min}$ before cannulated into the other test-tube. The final mixture was stirred at room temperature for $8 \mathrm{~h}$. Solvents were removed in vacuo and the regioselectivity was determined on the crude mixture by ${ }^{1} \mathrm{H}$ NMR spectroscopic methods. Column purification [petroleum ether-ether, 20:1 (v/v)] gave 19 (66mg, 71\%, 93\%ee) followed by $\mathbf{2 0}(14 \mathrm{mg}, 15 \%)$.

Data for 19: $\mathrm{R}_{\mathrm{f}}=0.14\left(20: 1\right.$ petroleum ether/ether). $[\alpha]^{25}{ }_{\mathrm{D}}+34.9\left(c=0.98, \mathrm{CH}_{2} \mathrm{Cl}_{2}\right.$, $78.1 \%$ ee, $(R, R)-\mathbf{L}-1]$. Enantiomers were separated via HPLC: chiralcel OC eluting with 99.5:0.5 heptane:isopropanol ( $0.75 \mathrm{ml} / \mathrm{min}$.), $\mathrm{t}_{\mathrm{r}}=36.00$ (minor), 39.36 (major). IR (film): 2226, 1624, 1592, 1507, 1466, 1443, $1410 \mathrm{~cm}^{-1}{ }^{1} \mathrm{H}$ NMR (300 MHz, $\left.\mathrm{CDCl}_{3}\right)$ ): 6.79$6.87(\mathrm{~m}, 4 \mathrm{H}), 5.99(\mathrm{~s}, 1 \mathrm{H}), 5.90(\mathrm{~s}, 1 \mathrm{H}), 4.54(\mathrm{t}, \mathrm{J}=6.3 \mathrm{~Hz}, 1 \mathrm{H}), 3.76(\mathrm{~s}, 3 \mathrm{H}), 1.76-$ $1.94(\mathrm{~m}, 2 \mathrm{H}), 1.40-1.64(\mathrm{~m}, 2 \mathrm{H}), 0.99(\mathrm{t}, \mathrm{J}=7.1 \mathrm{~Hz}, 3 \mathrm{H}) .{ }^{13} \mathrm{C} \mathrm{NMR}\left(75 \mathrm{MHz}, \mathrm{CDCl}_{3}\right)$

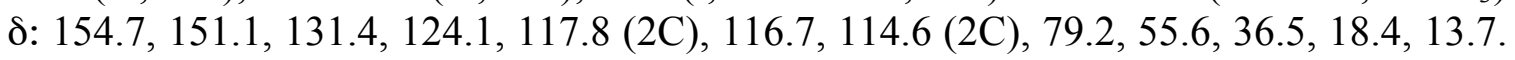
HRMS $\left(\mathrm{M}^{+}\right) \mathrm{C}_{14} \mathrm{H}_{17} \mathrm{NO}_{2}$. Calculated: 231.1259. Found: 231.1257.

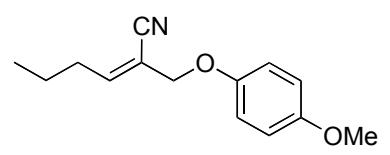

Compound 20: IR (film): 2226, 1624, 1592, 1507, 1466, $1410 \mathrm{~cm}^{-1} .{ }^{1} \mathrm{H}$ NMR (300 $\left.\mathrm{MHz}, \mathrm{CDCl}_{3}\right): \delta 6.85(\mathrm{~m}, 4 \mathrm{H}), 6.52(\mathrm{t}, \mathrm{J}=7.6 \mathrm{~Hz}, 1 \mathrm{H}), 4.53(\mathrm{~s}, 2 \mathrm{H}), 3.77(\mathrm{~s}, 3 \mathrm{H}), 2.41(\mathrm{q}$, $\mathrm{J}=7.3 \mathrm{~Hz}, 2 \mathrm{H}), 1.50(\mathrm{q}, \mathrm{J}=7.3 \mathrm{~Hz}, 2 \mathrm{H}), 0.95(\mathrm{t}, \mathrm{J}=7.3 \mathrm{~Hz}, 3 \mathrm{H}) .{ }^{13} \mathrm{CNMR}(75 \mathrm{MHz}$, $\left.\mathrm{CDCl}_{3}\right): \delta 154.5,151.0,129.0,128.4,116.3,114.6,111.4,68.8,56.7,33.3,21.6,13.5$. HRMS $\left(\mathrm{M}^{+}\right) \mathrm{C}_{14} \mathrm{H}_{17} \mathrm{NO}_{2}$. Calculated: 231.1259. Found: 231.1257. 


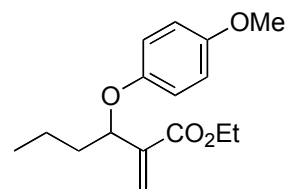

Compound 22: $p$-Methoxyphenol (34 mg, $0.28 \mathrm{mmol}),(S, S)-\mathbf{L}-3$ (5.8 $\mathrm{mg}, 8.3 \mu \mathrm{mol})$ and $\mathrm{Pd}_{2} \mathrm{dba}_{3} \cdot \mathrm{CHCl}_{3}(2.9 \mathrm{mg}, 2.8 \mu \mathrm{mol})$ were weighed into a test-tube directly. The test-tube was put under vacuum and refilled with argon (repeated 3 times). Allylic carbonate $\mathbf{2 1}^{7}$ (64 mg, $0.28 \mathrm{mmol}$ ) was weighed into another test-tube and methylene chloride $(2.8 \mathrm{~mL})$ was added. The solution was purged with argon for $15 \mathrm{~min}$ before cannulated into the other test-tube. The final mixture was stirred at room temperature for $8 \mathrm{~h}$. Solvents were removed in vacuo and the regioselectivity was determined on the crude mixture by ${ }^{1} \mathrm{H}$ NMR spectroscopic methods. Column purification [petroleum ether-ether, 20:1 (v/v)] gave 22 (49 mg, 64\%, 92\% ee) followed by 23 (18 $\mathrm{mg}, 24 \%$ ).

Data for 22: $\mathrm{R}_{\mathrm{f}}=0.27$ (25:1 petroleum ether/ether). $[\alpha]^{23} \mathrm{D}-21.7\left(c=0.75, \mathrm{CH}_{2} \mathrm{Cl}_{2}\right.$, $89.8 \%$ ee, $(R, R)$-L-1). Enantiomers were separated via HPLC: chiralcel OD eluting with 99.9:0.1 heptane:isopropanol (1.0 ml/min.); $\mathrm{t}_{\mathrm{r}}=13.89$ (major), 15.68 (minor). IR (film):

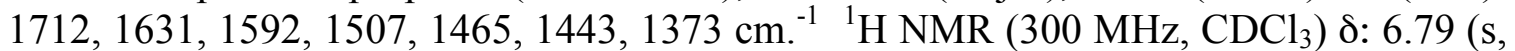
4H), $6.26(\mathrm{~s}, 1 \mathrm{H}), 5.84(\mathrm{~s}, 1 \mathrm{H}), 4.99-5.03(\mathrm{~m}, 1 \mathrm{H}), 4.26(\mathrm{q}, \mathrm{J}=7.1 \mathrm{~Hz}, 2 \mathrm{H}), 3.74(\mathrm{~s}, 3 \mathrm{H})$, 1.65-1.78 (m, 2H), 1.39-1.63 (m, 2H), $1.33(\mathrm{t}, \mathrm{J}=7.1 \mathrm{~Hz}, 3 \mathrm{H}), 0.95(\mathrm{t}, \mathrm{J}=7.3 \mathrm{~Hz}, 3 \mathrm{H})$. ${ }^{13} \mathrm{C}$ NMR $\left(75 \mathrm{MHz}, \mathrm{CDCl}_{3}\right)$ \&: 166.0, 153.7, 151.9, 140.7, 125.2, 116.3 (2C), 114.5 (2C), 75.7, 60.8, 55.6, 38.3, 18.9, 14.1, 13.8. HRMS $\left(\mathrm{M}^{+}\right) \mathrm{C}_{16} \mathrm{H}_{22} \mathrm{O}_{4}$. Calculated: 278.1518. Found: 278.1516.

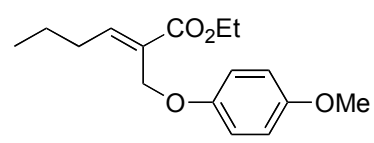

Compound 23: IR (film): 1714, 1622, 1508, 1466, 1399, $1367 \mathrm{~cm}^{-1} .{ }^{1} \mathrm{H}$ NMR (300

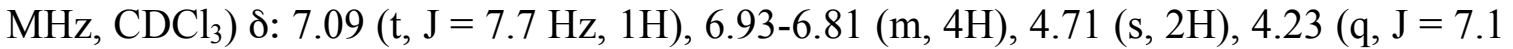
$\mathrm{Hz}, 2 \mathrm{H}), 3.76$ (s, 3H), 2.29 (q, J = 7.6 Hz, 2H), 1.50 (q, J = 7.3 Hz, 2H), 1.29(t, J = 7.1 $\mathrm{Hz}, 3 \mathrm{H}) .{ }^{13} \mathrm{C}$ NMR $\left(75 \mathrm{MHz}, \mathrm{CDCl}_{3}\right) \delta: 166.7,154.0,152.9,149.1,128.2,116.1(2 \mathrm{C})$, 114.5 (2C), 62.7, 60.7, 55.6, 30.8, 21.9, 14.2, 13.8. HRMS $\left(\mathrm{M}^{+}\right) \mathrm{C}_{16} \mathrm{H}_{22} \mathrm{O}_{4}$. Calculated: 278.1518. Found: 278.1516. 
Synthesis of $\mathbf{2 4}$ and 26:
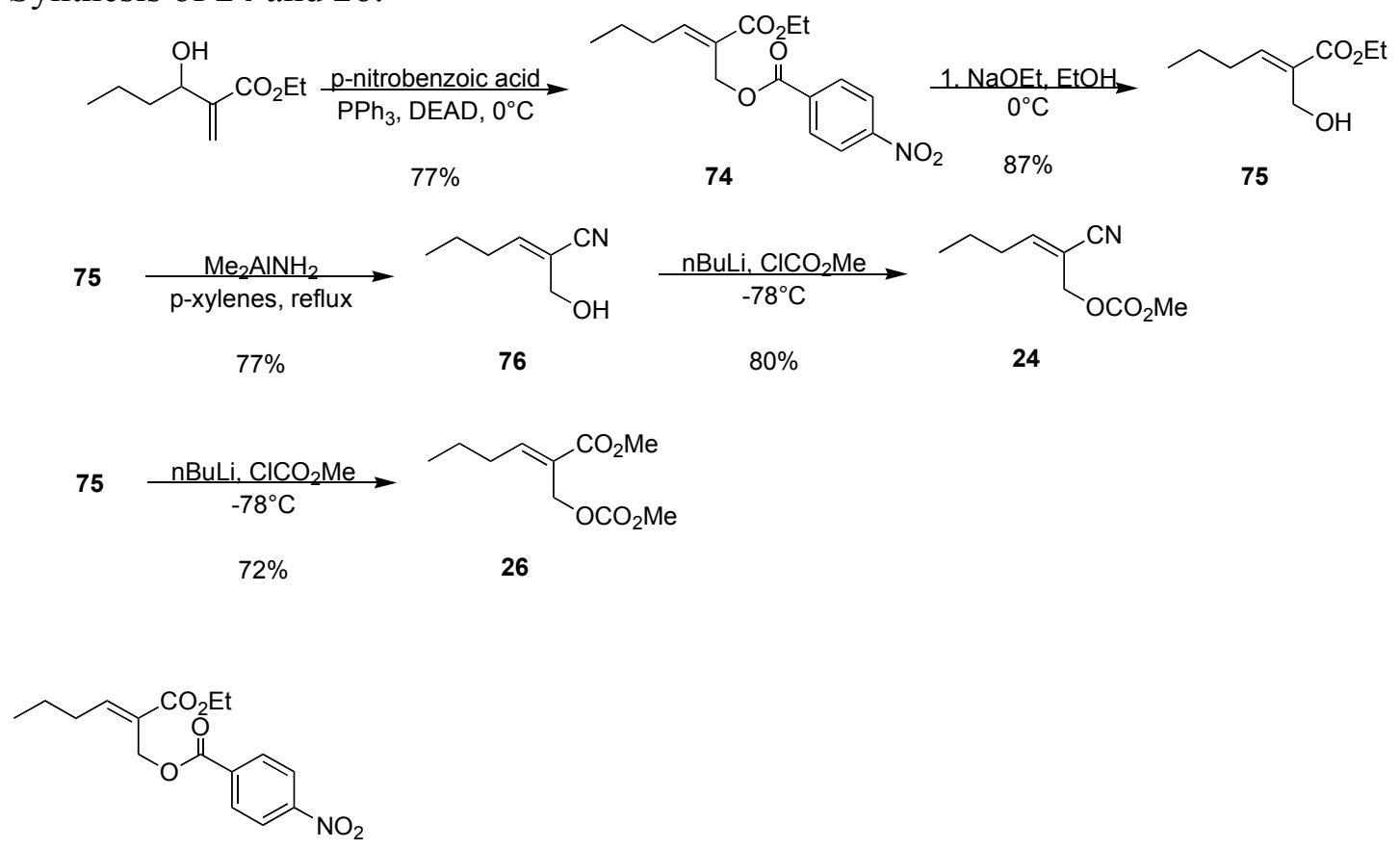

Compound 74: To a solution of triphenylphosphine $(2.28 \mathrm{~g}, 8.71 \mathrm{mmol})$ in tetrahydrofuran $(100 \mathrm{~mL})$ at $0^{\circ} \mathrm{C}$, diethyl azodicarboxylate $(1.37 \mathrm{ml}, 8.71 \mathrm{mmol})$ was added. After being stirred at room temperature for $10 \mathrm{~min}$, a solution of 2-(1-hydroxybutyl)-acrylic acid ethyl ester $(1.0 \mathrm{~g}, 5.81 \mathrm{mmol})$ in tetrahydrofuran $(10 \mathrm{~mL})$ was added dropwise. The mixture was stirred at room temperature for another $10 \mathrm{~min}$ before a solution of $p$-nitrobenzoic acid $(1.46 \mathrm{~g}, 8.71 \mathrm{mmol})$ in tetrahydrofuran $(20 \mathrm{~mL})$ was added. The final mixture was stirred at $0^{\circ} \mathrm{C}$ for a further $30 \mathrm{~min}$ before it was quenched with saturated sodium bicarbonate solution. Ether was added and layers were separated. The separated aqueous layer was extracted twice with ether. The combined organic layers were dried $\left(\mathrm{MgSO}_{4}\right)$ and filtered. Removal of solvents from the filtrate followed by column purification (7:1 petroleum ether/ether) gave ester $74(1.44 \mathrm{~g}, 77 \%)$ as colorless oil. $\mathrm{R}_{\mathrm{f}}=0.24$ (7:1 petroleum ether/ether) IR (film): 1725, 1650, 1608, 1530, $1464 \mathrm{~cm}^{-1}{ }^{1} \mathrm{H}$ NMR $\left(300 \mathrm{MHz}, \mathrm{CDCl}_{3}\right)$ d: $8.24(\mathrm{~d}, \mathrm{~J}=9.0 \mathrm{~Hz}, 2 \mathrm{H}), 8.15(\mathrm{~d}, \mathrm{~J}=9.0 \mathrm{~Hz}$, $2 \mathrm{H}), 7.13(\mathrm{t}, \mathrm{J}=7.8 \mathrm{~Hz}, 1 \mathrm{H}), 5.13(\mathrm{~s}, 2 \mathrm{H}), 4.23$ (q, J = $7.1 \mathrm{~Hz}, 2 \mathrm{H}), 2.34$ (q, J = $7.3 \mathrm{~Hz}$, 2H), 1.48-1.55 (m, 2H), $1.27(\mathrm{t}, \mathrm{J}=7.1 \mathrm{~Hz}, 3 \mathrm{H}), 0.94(\mathrm{t}, \mathrm{J}=7.3 \mathrm{~Hz}, 3 \mathrm{H}) .{ }^{13} \mathrm{C} \mathrm{NMR}(75$

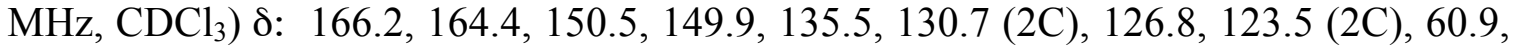
59.3, 30.7, 21.8, 14.2, 13.7. HRMS $\left(\mathrm{MH}^{+}\right) \mathrm{C}_{16} \mathrm{H}_{20} \mathrm{NO}_{6}$. Calculated: 322.1290. Found: 322.1294 .<smiles>CCCC=C(CO)C(=O)OCC</smiles>

Compound 75: To a solution of $74(351 \mathrm{mg}, 1.09 \mathrm{mmol})$ in ethanol $(5 \mathrm{ml})$ at $0^{\circ} \mathrm{C}$, sodium ethoxide $(7.4 \mathrm{mg}, 0.011 \mathrm{mmol})$ was added. After being stirred at $0^{\circ} \mathrm{C}$ for $2 \mathrm{hr}$., 
the reaction was quenched with saturated ammonium chloride solution. Ether was added and layers were separated. The separated aqueous layer was extracted twice with ether. The combined organic layers were dried $\left(\mathrm{MgSO}_{4}\right)$ and filtered. Removal of solvents from the filtrate followed by column purification (silica, 2:1 petroleum ether/ether, isochratic) gave $75(164 \mathrm{mg}, 87 \%)$ as colorless oil. $\mathrm{R}_{\mathrm{f}}=0.26$ (2:1 petroleum ether/ether). IR (film): 35101713, 1647, $1483 \mathrm{~cm}^{-1}{ }^{1} \mathrm{H}$ NMR (300 MHz, $\left.\mathrm{CDCl}_{3}\right) \delta: 6.84$ (t, J = 7.6 $\mathrm{Hz}, 1 \mathrm{H}), 4.29$ (s, 2H), 4.19 (q, J = 7.1 Hz, 2H), $2.68(\mathrm{~s}, 1 \mathrm{H}), 2.22$ (q, J = $7.6 \mathrm{~Hz}, 2 \mathrm{H})$, $1.40-1.52(\mathrm{~m}, 2 \mathrm{H}), 1.28(\mathrm{t}, \mathrm{J}=7.1 \mathrm{~Hz}, 3 \mathrm{H}), 0.91(\mathrm{t}, \mathrm{J}=7.3 \mathrm{~Hz}, 3 \mathrm{H}) .{ }^{13} \mathrm{C} \mathrm{NMR}(75 \mathrm{MHz}$, $\left.\mathrm{CDCl}_{3}\right) \delta: 167.5,145.3,131.1,60.6,57.1,30.2,21.9,14.1,13.7$.

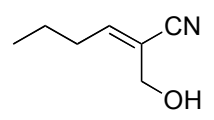

Compound 76: To a solution of alcohol 75 (344 mg, $2.00 \mathrm{mmol})$ in $p$-xylene $(40 \mathrm{~mL})$ at room temperature, a solution of dimethylaluminum amide $(6.7 \mathrm{ml}, 8.00 \mathrm{mmol}, 1.2 \mathrm{M})$ in dichloromethane was added. The mixture was heated to reflux for $3 \mathrm{~h}$. After being cooled to room temperature, the mixture was quenched with dilute sulfuric acid. Ether was added and layers were separated. The separated aqueous layer was extracted twice with ether. The combined organic layers were dried $\left(\mathrm{MgSO}_{4}\right)$ and filtered. Removal of solvents from the filtrate followed by column purification (silica, 1:1 petroleum ether/ether, isochratic) gave nitrile $76(194 \mathrm{mg}, 77 \%)$ as colorless oil. $\mathrm{R}_{\mathrm{f}}=0.28(1: 1$ petroleum ether/ether). IR (film): 3441, 2222, 1635, 1463, 964, $915 \mathrm{~cm}^{-1}{ }^{1} \mathrm{H}$ NMR (300 $\left.\mathrm{MHz}_{\mathrm{CDCl}}\right) \delta 6.48(\mathrm{t}, \mathrm{J}=7.8 \mathrm{~Hz}, 1 \mathrm{H}), 4.26(\mathrm{~s}, 2 \mathrm{H}), 2.50(\mathrm{~s}, 1 \mathrm{H}), 2.22(\mathrm{q}, \mathrm{J}=7.6 \mathrm{~Hz}$, $2 \mathrm{H}), 1.41-1.53(\mathrm{~m}, 2 \mathrm{H}), 0.93(\mathrm{t}, \mathrm{J}=7.6 \mathrm{~Hz}, 3 \mathrm{H}) .{ }^{13} \mathrm{C} \mathrm{NMR}\left(75 \mathrm{MHz}, \mathrm{CDCl}_{3}\right) \delta 150.5$, 119.0, 115.1, 57.7, 30.5, 21.7, 13.5.

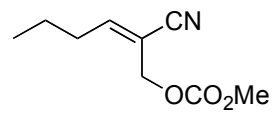

Compound 24: To a solution of alcohol 76 (124 $\mathrm{mg}, 0.99 \mathrm{mmol})$ in tetrahydrofuran (3 $\mathrm{ml})$ at $-78^{\circ} \mathrm{C}$ under argon, n-butyllithium $(0.62 \mathrm{ml}, 0.99 \mathrm{mmol}, 1.6 \mathrm{M})$ was added. After being stirred for $5 \mathrm{~min}$, chloromethyl formate $(0.11 \mathrm{ml}, 1.49 \mathrm{mmol})$ was added. The mixture was stirred at $-78^{\circ} \mathrm{C}$ for another 5 min before it was quenched with saturated ammonium chloride solution and warmed to room temperature. Ether was added and layers were separated. The separated aqueous layer was extracted twice with ether. The combined organic layers were dried $\left(\mathrm{MgSO}_{4}\right)$ and filtered. Removal of solvents from the filtrate followed by column purification (silica, 4:1 petroleum ether/ether, isochratic) gave carbonate $24(145 \mathrm{mg}, 80 \%)$ as colorless oil. $\mathrm{R}_{\mathrm{f}}=0.19$ (4:1 petroleum ether/ether). IR (film): 2223, 1755, 1639, 1445, $962 \mathrm{~cm}^{-1}{ }^{1} \mathrm{H} \mathrm{NMR}\left(300 \mathrm{MHz}, \mathrm{CDCl}_{3}\right)$ \&: 6.61 (t, J = $7.8 \mathrm{~Hz}, 1 \mathrm{H}), 4.71(\mathrm{~s}, 2 \mathrm{H}), 3.79$ (s, 3H), 2.27 (q, J = $7.6 \mathrm{~Hz}, 2 \mathrm{H}), 1.41-1.54(\mathrm{~m}, 2 \mathrm{H}), 0.92$ $(\mathrm{t}, \mathrm{J}=7.6 \mathrm{~Hz}, 3 \mathrm{H}) .{ }^{13} \mathrm{C}$ NMR $\left(75 \mathrm{MHz}, \mathrm{CDCl}_{3}\right) \delta$ 155.1, 153.9, 117.9, 109.9, 61.6, 55.1, 30.7, 21.5, 13.5. HRMS $\left(\mathrm{M}^{+}\right) \mathrm{C}_{9} \mathrm{H}_{13} \mathrm{NO}_{3}$. Calculated: 183.0895. Found: 183.0892. 


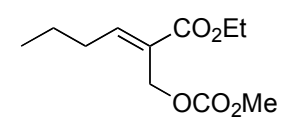

Compound 26: To a solution of $75(120 \mathrm{mg}, 0.70 \mathrm{mmol})$ in $5 \mathrm{~mL}$ THF at $-78^{\circ} \mathrm{C}$ under argon, n-butyllithium $(0.44 \mathrm{ml}, 0.70 \mathrm{mmol}, 1.6 \mathrm{M})$ was added. After being stirred for 5 min, chloromethyl formate $(0.08 \mathrm{ml}, 1.05 \mathrm{mmol})$ was added. The mixture was stirred at $-78^{\circ} \mathrm{C}$ for another $5 \mathrm{~min}$ before it was quenched with saturated ammonium chloride solution and warmed to room temperature. Ether was added and layers were separated. The separated aqueous layer was extracted twice with ether. The combined organic layers were dried $\left(\mathrm{MgSO}_{4}\right)$ and filtered. Removal of solvents from the filtrate followed by column purification (silica, 6:1 petroleum ether/ether, isochratic) gave carbonate 26 (116 mg, 72\%) as colorless oil. $\mathrm{R}_{\mathrm{f}}=0.24$ (6:1, petroleum ether/ether). IR (film): 1751, 1716, 1652, $1445 \mathrm{~cm}^{-1}{ }^{1} \mathrm{H}$ NMR $\left(300 \mathrm{MHz}, \mathrm{CDCl}_{3}\right) \delta 7.04(\mathrm{t}, \mathrm{J}=7.8 \mathrm{~Hz}, 1 \mathrm{H}), 4.88(\mathrm{~s}$, $2 \mathrm{H}), 4.18(\mathrm{q}, \mathrm{J}=7.1 \mathrm{~Hz}, 2 \mathrm{H}), 3.73(\mathrm{~s}, 3 \mathrm{H}), 2.27(\mathrm{q}, \mathrm{J}=7.6 \mathrm{~Hz}, 2 \mathrm{H}), 1.40-1.84(\mathrm{~m}, 2 \mathrm{H})$, $1.25(\mathrm{t}, \mathrm{J}=7.1 \mathrm{~Hz}, 3 \mathrm{H}), 0.91(\mathrm{t}, \mathrm{J}=7.6 \mathrm{~Hz}, 3 \mathrm{H}) .{ }^{13} \mathrm{C} \mathrm{NMR}\left(75 \mathrm{MHz}, \mathrm{CDCl}_{3}\right) \delta 166.1$, $155.5,149.8,126.7,61.2,60.7,54.7,30.6,21.8,14.1,13.7$.

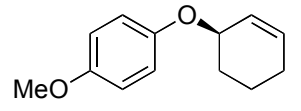

Compound 29: A test tube containing 4-methoxyphenol (146 mg, $1.18 \mathrm{mmol}$ ), $\mathrm{Pd}_{2} \mathrm{dba}_{3} \cdot \mathrm{CHCl}_{3}(12 \mathrm{mg}, 0.012 \mathrm{mmol}),(S, S)-\mathbf{L}-3$ (24 mg, $\left.0.035 \mathrm{mmol}\right)$ was evacuated and filled with argon three times. The test tube was charged with methylene chloride $(1.0 \mathrm{~mL})$ followed by addition of a solution of $28(184 \mathrm{mg}, 1.18 \mathrm{mmol})$ in methylene chloride (1.5 $\mathrm{mL})$. The resulting orange solution was stirred at $0^{\circ} \mathrm{C}$ for $8 \mathrm{~h}$ and directly chromatographed eluting with 5:1 petroleum ether:diethyl ether to afford $29(212 \mathrm{mg}$, $88 \%, 97 \%$ ee) as a colorless film. $[\alpha]_{\mathrm{D}}+129.1\left(\mathrm{c}=2.0, \mathrm{CHCl}_{3}\right)$. Enantiomers were separated by HPLC using Chiralcel OD column eluting with 99.5:0.5 heptanes:isopropanol at $1.0 \mathrm{~mL} / \mathrm{min}$. Retention times: minor enantiomer $(S) 11.51 \mathrm{~min}$. and major enantiomer $(R) 9.04 \mathrm{~min}$. The obtained spectral data matched literature values. ${ }^{8}$ IR (film): 1504, 1464, 1441, 956, $826 \mathrm{~cm}^{-1} .{ }^{1} \mathrm{H}$ NMR $\left(\mathrm{CDCl}_{3}, 300 \mathrm{MHz}\right): \delta 6.85$ $(\mathrm{m}, 4 \mathrm{H}), 5.96(\mathrm{dm}, \mathrm{J}=9.3 \mathrm{~Hz}, 1 \mathrm{H}), 5.87(\mathrm{~m}, 1 \mathrm{H}), 4.67(\mathrm{~m}, 1 \mathrm{H}), 3.77(\mathrm{~s}, 3 \mathrm{H}), 2.2-2.0(\mathrm{~m}$, $2 \mathrm{H}), 1.86(\mathrm{~m}, 3 \mathrm{H}), 1.63(\mathrm{~m}, 1 \mathrm{H}) .{ }^{13} \mathrm{C} \mathrm{NMR}\left(\mathrm{CDCl}_{3}, 75 \mathrm{MHz}\right): \delta 154.0,152.0,131.9$, 126.7, 117.4, 114.7, 71.9, 55.6, 28.2, 25.0, 18.9. Compound 29 has been made previously by this route. The absolute stereochemistry has also been proven. ${ }^{9}$ 
Synthesis of 30:

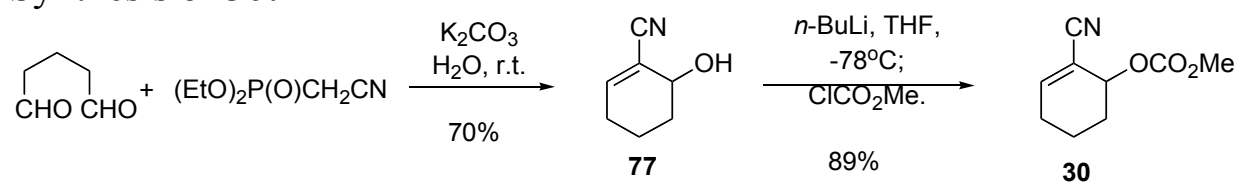<smiles>N#CC1=CCCCC1O</smiles>

Compound 77: The reaction was performed in analogy to a literature procedure. ${ }^{10}$ To a solution of glutaraldehyde $(3.4 \mathrm{~g}, 8.47 \mathrm{mmol}, 25 \%$ in water $)$ and diethyl (cyanomethyl)phosphate $(0.91 \mathrm{ml}, 5.65 \mathrm{mmol})$ at room temperature, a solution of potassium carbonate $(1.76 \mathrm{~g}, 12.71 \mathrm{mmol})$ in water $(2 \mathrm{ml})$ was added dropwise. The mixture was stirred at room temperature for $10 \mathrm{~min}$ before ether was added. Layers were separated and the separated aqueous layer was extracted twice with ether and dried $\left(\mathrm{MgSO}_{4}\right)$. Solvents were removed in vacuum and purification via chromatography (silica, 1:2 petroleum ether:ether, isochratic) gave alcohol $77(487 \mathrm{mg}, 70 \%)$ as colorless oil. $R_{\mathrm{f}}=0.41$ (1:2 petroleum ether:ether). IR (film): $3424,2222,1633,1452,1423 \mathrm{~cm}^{-1}$. ${ }^{1} \mathrm{H}$ NMR $\left(300 \mathrm{MHz}, \mathrm{CDCl}_{3}\right) \delta 6.72(\mathrm{t}, \mathrm{J}=4.0 \mathrm{~Hz}, 1 \mathrm{H}), 4.26-4.28(\mathrm{~m}, 1 \mathrm{H}), 2.99(\mathrm{~s}, 1 \mathrm{H})$, 2.06-2.30 (m, 2H), 1.54-1.92 (m, 4H). $\left.{ }^{13} \mathrm{C} \mathrm{NMR} \mathrm{(75} \mathrm{MHz,} \mathrm{CDCl}_{3}\right) \delta 147.8,118.5$, $116.5,64.4,30.5,25.9,17.3$.<smiles>COC(=O)C1CCCC=C1C#N</smiles>

Compound 30: To a solution of alcohol 77 (244 mg, $1.98 \mathrm{mmol})$ in tetrahydrofuran (3 $\mathrm{ml})$ at $-78^{\circ} \mathrm{C}$ under argon, n-butyllithium $(1.24 \mathrm{ml}, 1.98 \mathrm{mmol}, 1.6 \mathrm{M})$ was added. The anion mixture was stirred for $5 \mathrm{~min}$, at which time chloromethyl formate $(0.23 \mathrm{ml}, 2.97$ mmol) was added. The mixture was stirred at $-78^{\circ} \mathrm{C}$ for another $5 \mathrm{~min}$ before it was quenched with saturated ammonium chloride solution and warmed to room temperature. Ether was added and layers were separated. The separated aqueous layer was extracted twice with ether. The combined organic layers were dried $\left(\mathrm{MgSO}_{4}\right)$ and filtered. Removal of solvents from the filtrate followed by purification via chromatography (silica, 1:1 petroleum ether:ether, isochratic) gave $30(280 \mathrm{mg}, 78 \%)$ as colorless oil. $\mathrm{R}_{\mathrm{f}}=0.42$ (1:1 petroleum ether:ether). IR (film): 2221, 1749, 1637, 1581, 1443, $1424 \mathrm{~cm}^{-1}{ }^{1} \mathrm{H}$ NMR (300 MHz, CDCl $)$ d $6.86(\mathrm{t}, \mathrm{J}=3.9 \mathrm{~Hz}, 1 \mathrm{H}), 5.21(\mathrm{t}, \mathrm{J}=4.5 \mathrm{~Hz}, 1 \mathrm{H}), 3.77(\mathrm{~s}, 3 \mathrm{H})$, 2.24-2.34 (m, 1H), 2.10-2.22 (m, 1H), 1.82-1.94 (m, 2H), 1.61-1.79 (m, 2H). ${ }^{13} \mathrm{C} \mathrm{NMR}$ $\left(75 \mathrm{MHz}, \mathrm{CDCl}_{3}\right) \delta 154.6,150.6,117.2,111.9,69.5,54.9,27.3,25.6,16.5$. HRMS for $\mathrm{C}_{9} \mathrm{H}_{11} \mathrm{NO}_{3}\left(\mathrm{M}^{+}\right)$calcd 181.0739, obsd 181.0739. 


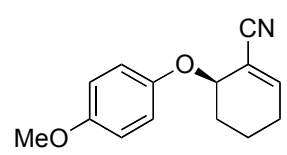

Compound 31: A test tube containing 4-methoxyphenol (45 $\mathrm{mg}, 0.37 \mathrm{mmol}$ ), $\mathrm{Pd}_{2} \mathrm{dba}_{3} \cdot \mathrm{CHCl}_{3}$ (3.8 $\left.\mathrm{mg}, 0.004 \mathrm{mmol}\right),(S, S)-\mathbf{L}-3$ (7.7 mg, $\left.0.012 \mathrm{mmol}\right)$ was evacuated and filled with argon three times. The test tube was charged with a solution of $\mathbf{3 0}(66 \mathrm{mg}$, $0.37 \mathrm{mmol}$ ) in methylene chloride $(3.7 \mathrm{~mL})$. The resulting orange solution was stirred at $0^{\circ} \mathrm{C}$ for $3 \mathrm{~h}$ and directly chromatographed eluting with $6: 1$ petroleum ether:diethyl ether to afford 31 (67 mg, 80\%, 40\%ee) as a colorless film. Enantiomers were separated by HPLC using Chiralcel OD column eluting with $95: 5$ heptanes:isopropanol at $1.0 \mathrm{~mL} / \mathrm{min}$. Retention times: minor enantiomer $(S) 37.20 \mathrm{~min}$. and major enantiomer $(R) 41.86 \mathrm{~min}$. IR (film): 2221, 1749, 1595, $1442 \mathrm{~cm}^{-1} .{ }^{1} \mathrm{H}$ NMR $\left(300 \mathrm{MHz}, \mathrm{CDCl}_{3}\right) \delta$ 6.80-7.02 (m, $4 \mathrm{H}), 6.82(\mathrm{br} \mathrm{s}, 1 \mathrm{H}), 3.77(\mathrm{~s}, 3 \mathrm{H}), 2.31-2.41(\mathrm{~m}, 1 \mathrm{H}), 2.12-2.30(\mathrm{~m}, 1 \mathrm{H}), 1.92-2.06(\mathrm{~m}$, $1 \mathrm{H}), 1.61-1.90(\mathrm{~m}, 4 \mathrm{H}) .{ }^{13} \mathrm{C} \mathrm{NMR}\left(75 \mathrm{MHz}, \mathrm{CDCl}_{3}\right) \delta 154.8,151.4,150.6,149.1,118.6$ (2C), $114.6(2 \mathrm{C}), 113.9,72.2,55.5,26.9,25.9,16.5 \mathrm{HRMS}$ for $\mathrm{C}_{14} \mathrm{H}_{15} \mathrm{NO}_{2}\left(\mathrm{M}^{+}\right)$Calc'd 229.1103. Found 229.1092. $[\alpha]_{\mathrm{D}}^{22}-48.2\left(c=0.78, \mathrm{CH}_{2} \mathrm{Cl}_{2}, 48 \% \mathrm{ee},(R, R)-\mathbf{L}-\mathbf{1}\right)$.

Stereochemical determination for $\mathbf{3 1}$ :

The stereochemistry was determined through removal of the PMP group and formation of the mandelate ester. ${ }^{11}$

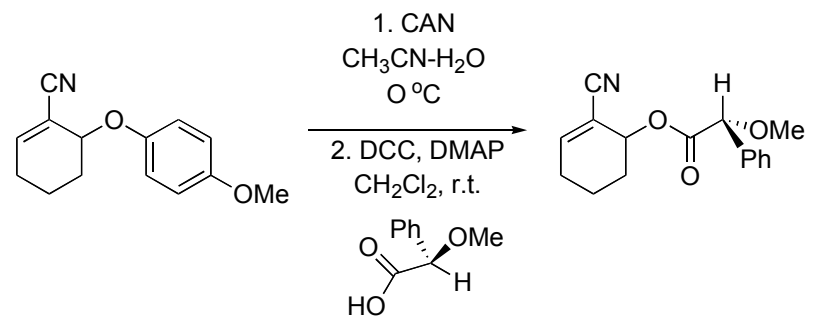

A solution of ceric ammonium nitrate $(538 \mathrm{mg}, 0.98 \mathrm{mmol})$ in water $(3 \mathrm{ml})$-acetonitrile (3 $\mathrm{ml}$ ) mixture was added dropwise to a solution of $\mathbf{3 1}(75 \mathrm{mg}, 0.33 \mathrm{mmol})$ in acetonitrile (2 $\mathrm{ml}$ ) at room temperature. The mixture was stirred at room temperature for $10 \mathrm{~min}$ at which time ether was added. The mixture was washed successively with saturated sodium bicarbonate, saturated sodium bisulfite solution, and brine. The separated organic layer was dried $\left(\mathrm{MgSO}_{4}\right)$, filtered, and solvents were removed in vacuum. Purification via chromatography (silica, 3:1 petroleum ether:ether, isochratic) gave an intermediate alcohol (28 $\mathrm{mg}, 70 \%)$ as colorless oil. A mixture of this alcohol (28 $\mathrm{mg}, 0.23 \mathrm{mmol})$, DCC (47 mg, $0.23 \mathrm{mmol})$ and $(R)$-O-methylmandelic acid $(41 \mathrm{mg}, 0.25 \mathrm{mmol})$ were dissolved in dichloromethane $(3 \mathrm{ml})$ at room temperature. DMAP $(2.8 \mathrm{mg}, 0.023 \mathrm{mmol})$ was added and the white suspension was stirred at room temperature overnight. The white solid was filtered and washed with ether. The organic layer was washed with aqueous hydrochloride acid (1\%), followed by saturated sodium bicarbonate solution. The organic layer was dried $\left(\mathrm{MgSO}_{4}\right)$, filtered and the volatile solvents were removed in vacuum. Purification via chromatography (silica, 1:1 petroleum ether:ether, isochratic) gave the mandelate ester $(48 \mathrm{mg}, 78 \%)$ as colorless oil. $\mathrm{R}_{\mathrm{f}}=0.50(1: 2$ petroleum ether:ether). IR (film): 2222, 1749, 1672, 1638, 1603, 1567, 1495, $1455 \mathrm{~cm}^{-1}{ }^{1} \mathrm{H}$ NMR $\left(300 \mathrm{MHz}, \mathrm{CDCl}_{3}\right) \delta$ 7.26-7.47 (m, 5H), 6.82-6.87 (m, 1H), 5.40-5.44 (m, 1H), $4.81(\mathrm{~s}$, $1 \mathrm{H}), 3.44(\mathrm{~s}, 3 \mathrm{H}), 2.07-2.26(\mathrm{~m}, 2 \mathrm{H}), 1.40-1.92(\mathrm{~m}, 4 \mathrm{H}) .{ }^{13} \mathrm{C}$ NMR $(75 \mathrm{MHz}$, 
$\left.\mathrm{CDCl}_{3}\right) \delta 170.0,150.4,135.9,128.8,128.6(2 \mathrm{C}), 127.1(2 \mathrm{C}), 117.3,112.3,82.2,66.7$, $57.4,27.0,25.6,16.7$.

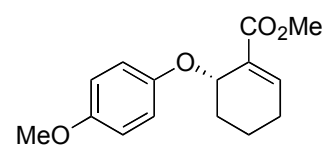

Compound 33: A test tube containing 4-methoxyphenol (15 mg, $0.121 \mathrm{mmol})$, $\mathrm{Pd}_{2} \mathrm{dba}_{3} \cdot \mathrm{CHCl}_{3}(1.0 \mathrm{mg}, 0.001 \mathrm{mmol}),(S, S)-\mathbf{L}-3$ (2 $\left.\mathrm{mg}, 0.003 \mathrm{mmol}\right)$ was evacuated and filled with argon three times. The test tube was charged with a solution of $\mathbf{3 2}(25 \mathrm{mg}$, $0.117 \mathrm{mmol})$ in methylene chloride $(1.0 \mathrm{~mL})$. The resulting orange solution was stirred at $0^{\circ} \mathrm{C}$ for $3 \mathrm{~h}$ and directly chromatographed eluting with $6: 1$ petroleum ether:diethyl ether to afford $33(24 \mathrm{mg}, 78 \%, 86 \% \mathrm{ee})$ as a colorless film. Enantiomers were separated by HPLC using Chiralcel OD column eluting with 98:2 heptanes:isopropanol at $1.0 \mathrm{~mL} / \mathrm{min}$. Retention times: minor enantiomer $(R) 8.38 \mathrm{~min}$. and major enantiomer $(S) 10.25 \mathrm{~min}$. IR (film): 1717, 1506, $1436 \mathrm{~cm}^{-1} .{ }^{1} \mathrm{H}$ NMR $\left(\mathrm{CDCl}_{3}, 500 \mathrm{MHz}\right): \delta 7.24(\mathrm{~m}, 1 \mathrm{H}), 6.99(\mathrm{dm}, \mathrm{J}$ $=9.0 \mathrm{~Hz}, 2 \mathrm{H}), 6.81(\mathrm{dm}, \mathrm{J}=9.0 \mathrm{~Hz}, 2 \mathrm{H}), 5.01(\mathrm{~m}, 1 \mathrm{H}), 3.76(\mathrm{~s}, 3 \mathrm{H}), 3.74(\mathrm{~s}, 3 \mathrm{H}), 2.38(\mathrm{dt}$, $\mathrm{J}=19.8,4.6 \mathrm{~Hz}, 1 \mathrm{H}), 2.20-2.09(\mathrm{~m}, 2 \mathrm{H}), 1.89-1.79(\mathrm{~m}, 1 \mathrm{H}), 1.66-1.60(\mathrm{~m}, 1 \mathrm{H}), 1.43$ (tt, J $=14.0,3.4 \mathrm{~Hz}, 1 \mathrm{H}) .{ }^{13} \mathrm{C} \mathrm{NMR}\left(\mathrm{CDCl}_{3}, 125 \mathrm{MHz}\right): \delta 166.9,154.4,152.0,144.8,129.7$, 118.9 (2C), 114.5 (2C), 70.1, 55.6, 51.7, 26.3, 25.9, 15.8. HRMS Calc'd for $\mathrm{C}_{15} \mathrm{H}_{18} \mathrm{O}_{4}$ : 262.1205. Found 262.1198. The absolute stereochemistry of $\mathbf{3 3}$ was determined in analogy to that seen in the galanthamine synthesis. ${ }^{12}$<smiles>C=C(C(=O)O)C1CCC(=CC)CO1</smiles>

Compound 34. Saponification: $\mathrm{LiOH}(98 \mathrm{mg}$ in $1.2 \mathrm{~mL}$ water) was added dropwise over $1 \mathrm{~h}$ via syringe pump to a cooled $\left(0^{\circ} \mathrm{C}\right)$ solution of $15(0.030 \mathrm{~g}, 0.15 \mathrm{mmol})$ in THF $(1.5 \mathrm{~mL})$. After the addition was complete, the solution was warmed to room temperature and stirred for $20 \mathrm{~h}$. The resulting mixture was then acidified with $1 \mathrm{M} \mathrm{HCl}$ and diluted with ether. The aqueous layer was separated and extracted three times with ether. The combined extracts were washed with brine and dried over anhydrous magnesium sulfate, filtered, and concentrated in vacuo. Flash chromatography on deactivated silica $(10 \% \mathrm{wt}$ water on silica gel, hexane:EtOAc 4:1 isochratic) afforded $0.0256 \mathrm{~g}$ (94\%) of 34 as an oily white solid.

DIBAl-H reduction/Pinnick oxidation: DIBAl-H $(0.2 \mathrm{~mL}$ of $1.0 \mathrm{M}$ in toluene) was added to a cooled $\left(-78^{\circ} \mathrm{C}\right)$ solution of $17(0.03 \mathrm{~g}, 0.18 \mathrm{mmol})$ in $0.6 \mathrm{~mL}$ toluene. The resulting solution was stirred at $-78^{\circ} \mathrm{C}$ for $3 \mathrm{~h}$ at which time it was quenched via the addition of a $7 \%$ aqueous $\mathrm{NaH}_{2} \mathrm{SO}_{4}$ solution. The reaction was allowed to warm to $0^{\circ} \mathrm{C}$ and stirred at that temperature for $30 \mathrm{~min}$. The resulting aqueous layer was extracted three times with diethyl ether. The combined organic layers were dried over $\mathrm{MgSO}_{4}$, filtered, and concentrated in vacuo. The resulting oily residue was directly taken up in t-BuOH $(1.2$ $\mathrm{mL})$. 2-Methyl-2-butene $(0.4 \mathrm{~mL})$ was added, followed by a solution of $\mathrm{KH}_{2} \mathrm{PO}_{4}(0.12 \mathrm{~g}$, $0.87 \mathrm{mmol})$ and $\mathrm{NaClO}_{2}(0.085 \mathrm{~g}, 0.94 \mathrm{mmol})$ in $1.0 \mathrm{~mL} \mathrm{H}_{2} \mathrm{O}$. The resulting solution was stirred vigorously at room temperature for $3 \mathrm{~h}$. The reaction was poured into water and ether. The layers were separated and the aqueous layer was extracted three times with 
ethyl acetate. The combined organic layers were basified with $1 \mathrm{M} \mathrm{NaOH}$ and washed three times with water. The combined aqueous layers were acidified with conc. $\mathrm{HCl}$ and then extracted three times with ethyl acetate. The combined ethyl acetate layers were dried over $\mathrm{MgSO}_{4}$, filtered, and concentrated in vacuo to afford $0.013 \mathrm{~g}(40 \%)$ of spectroscopically pure $\mathbf{3 4}$ as an oily white solid. For material with $81 \%$ ee, $[\alpha]_{\mathrm{D}}+112.66$ (c = 0.82, $\mathrm{CH}_{2} \mathrm{Cl}_{2}$ ). IR (film from $\mathrm{CDCl}_{3}$ ): 2935(broad), 1694, 1628, $1435 \mathrm{~cm}^{-1} .{ }^{1} \mathrm{H}$ NMR $\left(400 \mathrm{MHz}, \mathrm{CDCl}_{3}\right) \delta 6.39(\mathrm{~s}, 1 \mathrm{H}), 5.97(\mathrm{~s}, 1 \mathrm{H}), 5.29(\operatorname{app~q}, \mathrm{J}=7.2 \mathrm{~Hz}, 1 \mathrm{H}), 4.74(\mathrm{~d}, \mathrm{~J}=$ $12.4 \mathrm{~Hz}, 1 \mathrm{H}), 4.30(\mathrm{~d}, \mathrm{~J}=11.2 \mathrm{~Hz}, 1 \mathrm{H}), 3.87(\mathrm{~d}, \mathrm{~J}=12.4 \mathrm{~Hz}, 1 \mathrm{H}), 2.33(\mathrm{~m}, 2 \mathrm{H}), 2.03(\mathrm{~m}$, $1 \mathrm{H}), 1.61(\mathrm{~d}, \mathrm{~J}=6.8 \mathrm{~Hz}, 3 \mathrm{H}), 1.38(\mathrm{~m}, 1 \mathrm{H})$. The carboxylic acid proton was too broad to be observed. ${ }^{13} \mathrm{C}$ NMR $\left(100 \mathrm{MHz}, \mathrm{CDCl}_{3}\right) \delta 170.7,140.7,133.2,127.3,119.3,75.5$, 66.7, 33.7, 32.9, 12.7. HRMS ( $\left.\mathrm{M}^{+}-\mathrm{H}\right) \mathrm{C}_{10} \mathrm{H}_{13} \mathrm{O}_{3}$. Calculated: 181.0865. Found: 181.0862.

\section{PART III. Data for (+)-hippospongic acid A synthesis:}<smiles>CC(=O)OC/C=C(\C)CCC=O</smiles>

Compound 44. The ozonylsis was performed in analogy to a literature procedure. ${ }^{13}$ Ozone was bubbled through a cooled $\left(-78^{\circ} \mathrm{C}\right)$ solution of geranyl acetate $(3.2 \mathrm{~mL}, 15$ $\mathrm{mmol})$, pyridine $(3.75 \mathrm{~mL})$ and DCM $(115 \mathrm{~mL})$. The reaction progress was monitored via TLC (every ten min a TLC was taken) until complete consumption of starting material was observed. At that time, the excess ozone was removed by bubbling argon through the solution. $\mathrm{PPh}_{3}(11.5 \mathrm{~g}, 44 \mathrm{mmol})$ was added and the reaction was warmed slowly to room temperature under argon over $4 \mathrm{~h}$. The resulting solution was concentrated in vacuo and purified directly via flash chromatography (silica, ether/petroleuym ether, gradient) to yield $1.67 \mathrm{~g}(66 \%)$ of 44 as a colorless oil. $\mathrm{R}_{\mathrm{f}}=0.2$ in $25 \%$ ether/petroleum ether. The obtained spectral data [IR, $\left.{ }^{1} \mathrm{H}\right]$ matched literature values. ${ }^{14}$ IR (neat): 2919, 2832, 2726, 1732, 1674, 1445, $1416 \mathrm{~cm}^{-1} .{ }^{1} \mathrm{H}$ NMR $\left(300 \mathrm{MHz}, \mathrm{CDCl}_{3}\right) \delta 9.79(\mathrm{~s}, 1 \mathrm{H}), 5.37$ (t, J = 7.0 $\mathrm{Hz}, 1 \mathrm{H}), 4.59$ (d, J = 7.2 Hz, 2H), 2.59 (t, J = 7.5 Hz, 2H), 2.39 (t, J = 7.5 Hz, 2H), 2.06 $(\mathrm{s}, 3 \mathrm{H}), 1.73(\mathrm{~s}, 3 \mathrm{H})$.

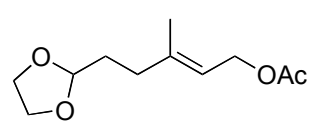

Compound 45. PPTS $(0.0025 \mathrm{~g}, 0.0099 \mathrm{~mol})$ was added to a solution of $44(0.20 \mathrm{~g}, 1.2$ $\mathrm{mmol})$ and ethylene glycol $(0.12 \mathrm{~mL}, 2.15 \mathrm{mmol})$ in benzene $(2.5 \mathrm{~mL})$. The reaction was heated to reflux under Dean Stark conditions for $3.5 \mathrm{~h}$. Note: It is important to monitor the conversion carefully as decomposition of product occurs under the reaction conditions if left too long. The cooled mixture was diluted with ether and washed with saturated $\mathrm{NaHCO}_{3}$. The aqueous layer was extracted twice with ether. The combined organic layers were dried over magnesium sulfate, and concentrated in vacuo. The resulting colorless oil $(0.1992 \mathrm{~g}, 77 \%$ of 45$)$ was used without further purification. The 
obtained spectral data $\left[\mathrm{IR},{ }^{1} \mathrm{H}\right]$ matched literature values ${ }^{15}$. IR (neat): $2956,2885,1445$ $\mathrm{cm}^{-1} .{ }^{1} \mathrm{H}$ NMR $\left(300 \mathrm{MHz}, \mathrm{CDCl}_{3}\right) \delta 5.28(\mathrm{dt}, \mathrm{J}=1.2,7.0 \mathrm{~Hz}, 1 \mathrm{H}), 4.77(\mathrm{t}, \mathrm{J}=4.8 \mathrm{~Hz}$, $1 \mathrm{H}), 5.49(\mathrm{~d}, \mathrm{~J}=7.0 \mathrm{~Hz}, 2 \mathrm{H}), 3.73-3.90(\mathrm{~m}, 4 \mathrm{H}), 2.07$ (m, 2H), $1.96(\mathrm{~s}, 3 \mathrm{H}), 1.73-1.66$ $(\mathrm{m}, 2 \mathrm{H}), 1.62(\mathrm{~s}, 3 \mathrm{H})$.

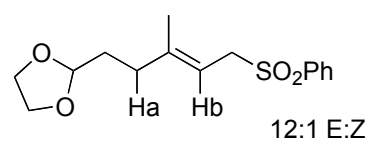

Compound 46. Note: Both the methylene chloride and water are both fully degassed with argon before starting the reaction. A solution of $(\mathrm{Pd}(\pi \text {-allyl }) \mathrm{Cl})_{2}(0.033 \mathrm{~g}, 0.09$ mmol $)$ and dppf $(0.15 \mathrm{~g}, 0.27 \mathrm{mmol})$ in $5 \mathrm{~mL} \mathrm{CH}_{2} \mathrm{Cl}_{2}$ was added to a flask containing $\mathrm{PhSO}_{2} \mathrm{Na}(1.26 \mathrm{~g}, 7.7 \mathrm{mmol})$, tetramethylammonium bromide $(0.063 \mathrm{~g}, 0.41 \mathrm{mmol})$, and $39(0.97 \mathrm{~g}, 4.51 \mathrm{mmol})$, in a mixture of water $(15 \mathrm{~mL})$ and $\mathrm{CH}_{2} \mathrm{Cl}_{2}(10 \mathrm{~mL})$. The reaction was stirred at room temperature for $2.5 \mathrm{~h}$, at which time the layers were separated. The aqueous layer was extracted 3 times with $\mathrm{CH}_{2} \mathrm{Cl}_{2}$, dried over magnesium sulfate, concentrated in vacuo, and purified via flash chromatography (silica, ether/petroleum ether, gradient) to yield $1.307 \mathrm{~g}(98 \%)$ of $\mathbf{4 6}$ as a colorless oil. The major isomer was determined to be trans via comparison to literature and via nOe studies $(500 \mathrm{MHz}$ in $\mathrm{CDCl}_{3}$ ). There was a $6.7 \%$ nOe between $\mathrm{Ha}$ and $\mathrm{Hb}$ in the major isomer and $0 \% \mathrm{nOe}$ between the corresponding hydrogens in the minor isomer. The obtained spectral data $\left[\mathrm{IR},{ }^{1} \mathrm{H}\right]$ for the major isomer matched the literature values ${ }^{4}$ : IR (neat): 1663,1447 , $1406 \mathrm{~cm}^{-1} .{ }^{1} \mathrm{H}$ NMR $\left(500 \mathrm{MHz}, \mathrm{CDCl}_{3}\right) \delta 7.85(\mathrm{~d}, \mathrm{~J}=7.5 \mathrm{~Hz}, 2 \mathrm{H}), 7.63(\mathrm{t}, \mathrm{J}=7.5 \mathrm{~Hz}$, $1 \mathrm{H}), 7.53(\mathrm{~m}, 2 \mathrm{H}), 5.21(\mathrm{t}, \mathrm{J}=8.0 \mathrm{~Hz}, 1 \mathrm{H}), 4.80(\mathrm{t}, \mathrm{J}=5.0 \mathrm{~Hz}, 1 \mathrm{H}), 3.94(\mathrm{~m}, 2 \mathrm{H}), 3.85-$ $3.79(\mathrm{~m}, 4 \mathrm{H}), 2.097(\mathrm{t}, \mathrm{J}=8.0 \mathrm{~Hz}, 2 \mathrm{H}), 1.66(\mathrm{~m}, 2 \mathrm{H}), 1.30(\mathrm{~s}, 3 \mathrm{H}) .{ }^{13} \mathrm{C}$ NMR $(125 \mathrm{MHz}$, $\left.\mathrm{CDCl}_{3}\right) \delta 145.6,138.4,133.6,129.0$ (2C), 128.5 (2C), 110.6, 103.7, 64.9, 56.0, 33.7, $31.8,31.5,16.1$.

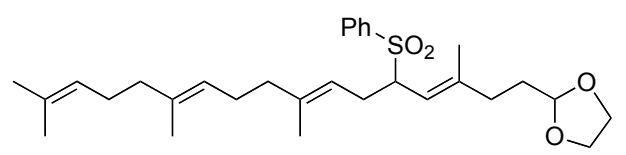

Compound 49. LDA (1.42 mL DIPA, $5.92 \mathrm{~mL}$ of $1.6 \mathrm{M} \mathrm{nBuLi}, 8 \mathrm{~mL}$ THF) was added to a cooled $\left(-78^{\circ} \mathrm{C}\right)$ solution of $46(1.88 \mathrm{~g}, 6.3 \mathrm{mmol})$ in $18 \mathrm{~mL}$ THF and $6 \mathrm{~mL}$ DMPU. The resulting bright orange solution was stirred for $30 \mathrm{~min}$ at $-78^{\circ} \mathrm{C}$, at which point farnesyl bromide $(1.8 \mathrm{~mL}, 6.6 \mathrm{mmol})$ was added dropwise. The bright orange color then changed to a light yellow color. The reaction was stirred an additional $10 \mathrm{~min}$ at $-78^{\circ} \mathrm{C}$, at which time it was quenched with saturated aqueous ammonium chloride. The aqueous layer was extracted three times with ether, dried over magnesium sulfate, and concentrated in vacuo. The resulting oily residue was purified via flash chromatography (silica, ether/petroleum ether, gradient) to yield $2.86 \mathrm{~g} \mathrm{(90 \% )} \mathrm{of} 49$ as a colorless oil. $\mathrm{R}_{\mathrm{f}}=$ 0.54 in $60 \%$ ether/petroelum ether, $\mathrm{KMnO}_{4}$ stain. The obtained spectral data $\left[\mathrm{IR},{ }^{1} \mathrm{H},{ }^{13} \mathrm{C}\right]$ matched the literature values ${ }^{16}$. IR (film from $\mathrm{CDCl}_{3}$ ): 1665, 1586, 1447, 1384, 894, 741, 
718, $690 \mathrm{~cm}^{-1} .{ }^{1} \mathrm{HNMR}\left(500 \mathrm{MHz}, \mathrm{CDCl}_{3}\right) \delta 7.87(\mathrm{~d}, \mathrm{~J}=7.0 \mathrm{~Hz}, 2 \mathrm{H}), 7.64(\mathrm{t}, \mathrm{J}=7.0 \mathrm{~Hz}$, $1 \mathrm{H}), 7.54(\mathrm{t}, \mathrm{J}=7.0 \mathrm{~Hz}, 2 \mathrm{H}), 5.07(\mathrm{~m}, 3 \mathrm{H}), 4.97(\mathrm{t}, \mathrm{J}=7.5 \mathrm{~Hz}, 1 \mathrm{H}), 4.81(\mathrm{t}, \mathrm{J}=4.5 \mathrm{~Hz}, 1 \mathrm{H})$, $3.97(\mathrm{~m}, 2 \mathrm{H}), 3.87(\mathrm{~m}, 2 \mathrm{H}), 3.75(\mathrm{td}, \mathrm{J}=10.0,3.0 \mathrm{~Hz}, 1 \mathrm{H}), 2.90(\mathrm{ddd}, \mathrm{J}=3.0,7.5,14.0 \mathrm{~Hz}$, $1 \mathrm{H}), 2.37$ (ddd, J=3.0, 7.5, 14.0Hz, 1H), 2.10-1.90 (m, 10H), $1.69(\mathrm{~s}, 3 \mathrm{H}), 1.67-1.63(\mathrm{~m}$, $2 \mathrm{H}), 1.62(\mathrm{~s}, 3 \mathrm{H}), 1.61(\mathrm{~s}, 3 \mathrm{H}), 1.59(\mathrm{~s}, 3 \mathrm{H}), 1.21(\mathrm{~d}, \mathrm{~J}=1.0 \mathrm{~Hz}, 3 \mathrm{H}) .{ }^{13} \mathrm{CNMR}(125 \mathrm{MHz}$, $\left.\mathrm{CDCl}_{3}\right) \delta 144.3,138.7,137.9,135.1,133.3,131.3,129.1$ (2C), $128.7(2 \mathrm{C}), 124.2,123.8$, $118.3,117.4,103.8,64.9$ (2C), 64.7, 39.7 (2C), 33.7, 31.9, 26.7, 26.5, 26.3, 25.7, 17.7, $16.4,16.4,15.9$.

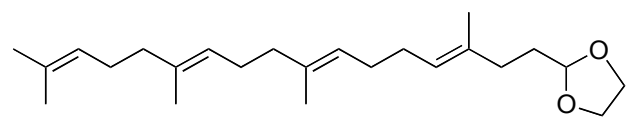

Compound 50. Procedure in analogy to literature. ${ }^{17}$ A slurry of $[\mathrm{Pd}(\pi \text {-allyl }) \mathrm{Cl}]_{2}(0.158$ g, $0.43 \mathrm{mmol})$ and dppp $(0.54 \mathrm{~g}, 1.3 \mathrm{mmol})$ in THF $(2 \mathrm{~mL})$ was added to $49(2.89 \mathrm{~g}, 5.77$ $\mathrm{mmol})$ in THF (56 mL) at room temperature, followed by the addition of $\mathrm{LiBHEt}_{3}(14.42$ $\mathrm{mL}$ of $1.0 \mathrm{M}$ in THF). After the addition the slurry became a yellow solution whose color quickly changes to red and finally to brown. The reaction was stirred at room temperature for $2.5 \mathrm{~h}$, at which time it was quenched with saturated aqueous $\mathrm{NH}_{4} \mathrm{Cl}$. The resulting aqueous layer was extracted with ether three times. The combined organic layers were washed with brine, dried over $\mathrm{MgSO}_{4}$, filtered, and concentrated in vacuo. The resulting oily residue was purified via flash chromatography (silica, ether/petroleum ether, gradient) to yield $1.95 \mathrm{~g}(94 \%)$ of $\mathbf{5 0}$ as a colorless oil. $\mathrm{R}_{\mathrm{f}}=0.9$ in $2: 1$ ether:petroleum ether, $\mathrm{KMnO}_{4}$ stain. The obtained spectral data [IR, ${ }^{1} \mathrm{H},{ }^{13} \mathrm{C}$ ] matched the literature values. ${ }^{16}$ IR (neat): 2925, 1448, $1382 \mathrm{~cm}^{-1} .{ }^{1} \mathrm{H} \mathrm{NMR}\left(500 \mathrm{MHz}, \mathrm{CDCl}_{3}\right) \delta 5.18(\mathrm{~m}, 1 \mathrm{H})$, $5.10(\mathrm{~m}, 3 \mathrm{H}), 4.85(\mathrm{t}, \mathrm{J}=4.5 \mathrm{~Hz}, 1 \mathrm{H}), 3.97(\mathrm{~m}, 2 \mathrm{H}), 3.85(\mathrm{~m}, 2 \mathrm{H}), 2.13-1.95(\mathrm{~m}, 14 \mathrm{H})$, $1.74(\mathrm{~m}, 2 \mathrm{H}), 1.68(\mathrm{~s}, 3 \mathrm{H}), 1.61(\mathrm{~s}, 3 \mathrm{H}), 1.60\left(\right.$ broad s, 9H). ${ }^{13} \mathrm{C} \mathrm{NMR}\left(125 \mathrm{MHz}, \mathrm{CDCl}_{3}\right)$ $\delta 135.1,134.8,134.2,131.2,124.5,124.4,124.2,124.1,104.3,64.8$ (2C), 39.7 (2C), $33.9,32.4,28.2,28.1,26.7,26.6,25.7,17.6,16.0$ (3C). Anal calcd for $\mathrm{C}_{24} \mathrm{H}_{40} \mathrm{O}_{2}$ : C, 79.94\%; H, 11.18\%. Found: C, 80.18\%; H, 10.98\%.

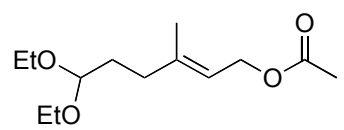

Compound 52. A solution of 37 (3.99 g, $23.44 \mathrm{mmol})$, ethanol (15 mL, $300 \mathrm{mmol})$ and benzene $(30 \mathrm{~mL})$ was added to a flask containing pyridinium $p$-toluenesulfonic acid $(0.041 \mathrm{~g}, 0.16 \mathrm{mmol})$. The reaction was heated to reflux under Dean Stark conditions for $1 \mathrm{~h}$. After completion, the cooled mixture was diluted with ether and washed with saturated sodium bicarbonate. The organic layer was dried over $\mathrm{MgSO}_{4}$, filtered, and concentrated in vacuo. The resulting oily residue was purified via flash chromatography (silica, ether/petroleum ether, gradient) to yield $5.13 \mathrm{~g}(90 \%)$ of $\mathbf{5 2}$ as a colorless oil. $\mathrm{R}_{\mathrm{f}}=$ 0.59 in 1:1 petroleum ether:diethyl ether, $\mathrm{KMnO}_{4}$ stain. IR (neat): $1741,1445 \mathrm{~cm}^{-1} .{ }^{1} \mathrm{H}$ NMR (400 MHz, $\left.\mathrm{CDCl}_{3}\right) \delta 5.32(\mathrm{t}, \mathrm{J}=6.8 \mathrm{~Hz}, 1 \mathrm{H}), 4.54(\mathrm{~d}, \mathrm{~J}=6.8 \mathrm{~Hz}, 2 \mathrm{H}), 4.22(\mathrm{t}, \mathrm{J}=$ 
$5.6 \mathrm{~Hz}, 1 \mathrm{H}), 3.60(\mathrm{~m}, 2 \mathrm{H}), 3.45(\mathrm{~m}, 2 \mathrm{H}), 2.06(\mathrm{~m}, 2 \mathrm{H}), 2.01(\mathrm{~s}, 3 \mathrm{H}), 1.71(\mathrm{~m}, 2 \mathrm{H}), 1.67$ $(\mathrm{s}, 3 \mathrm{H}), 1.16(\mathrm{t}, \mathrm{J}=7.2 \mathrm{~Hz}, 6 \mathrm{H}) .{ }^{13} \mathrm{C}$ NMR $\left(125 \mathrm{MHz}, \mathrm{CDCl}_{3}\right) \delta 171.09,141.65,118.36$, $102.36,102.38,61.29,60.99,34.48,31.47,21.02,16.47,15.32$ (2C). Anal calcd for $\mathrm{C}_{13} \mathrm{H}_{24} \mathrm{O}_{4}: \mathrm{C}, 63.91 \%$; H, 9.90\%. Found: C, 63.70\%; H, 9.77\%.

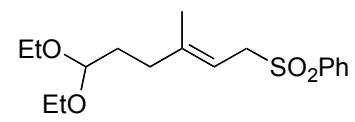

Compound 53. Note: Both the methylene chloride and water are both fully degassed with argon before starting the reaction. A solution of $(\mathrm{Pd}(\pi \text {-allyl }) \mathrm{Cl})_{2}(0.153 \mathrm{~g}, 0.42$ mmol $)$ and dppf $(0.698 \mathrm{~g}, 1.26 \mathrm{mmol})$ in $10 \mathrm{~mL} \mathrm{CH} \mathrm{Cl}_{2}$ is added via cannula to a flask containing $\mathrm{PhSO}_{2} \mathrm{Na}(5.86 \mathrm{~g}, 35.7 \mathrm{mmol})$, tetramethylammonium bromide $(0.291 \mathrm{~g}, 1.9$ $\mathrm{mmol})$, and $52(5.13 \mathrm{~g}, 20.99 \mathrm{mmol})$, in water $(70 \mathrm{~mL})$ and $\mathrm{CH}_{2} \mathrm{Cl}_{2}(60 \mathrm{~mL})$. The reaction was stirred at room temperature for $1.5 \mathrm{~h}$, at which time the layers were separated. The aqueous layer was extracted three times with $\mathrm{CH}_{2} \mathrm{Cl}_{2}$, dried over magnesium sulfate, and concentrated in vacuo. The resulting oily residue was purified via flash chromatography (silica, ether/petroleum ether) to yield 7.1 g (Q) of $\mathbf{5 3}$ as a colorless oil. This compound was isolated as a 95:5 mixture of trans to cis olefin geometries. The spectral data for the major isomer follows. IR (neat): $1664 \mathrm{~cm}^{-1} .{ }^{1} \mathrm{H} \mathrm{NMR}\left(500 \mathrm{MHz}, \mathrm{CDCl}_{3}\right) \delta 7.83(\mathrm{~m}, 2 \mathrm{H})$, $7.61(\mathrm{~m}, 1 \mathrm{H}), 7.51(\mathrm{~m}, 2 \mathrm{H}), 5.19(\mathrm{t}, \mathrm{J}=8.0 \mathrm{~Hz}, 1 \mathrm{H}), 4.39$ (t, J = 6.0 Hz, 1H), 3.77 (d, J = $8.0 \mathrm{~Hz}, 2 \mathrm{H}), 3.58(\mathrm{~m}, 2 \mathrm{H}), 3.45(\mathrm{~m}, 2 \mathrm{H}), 2.02(\mathrm{~m}, 2 \mathrm{H}), 1.61(\mathrm{~m}, 2 \mathrm{H}), 1.23(\mathrm{~s}, 3 \mathrm{H}), 1.17$ $(\mathrm{t}, \mathrm{J}=7.0 \mathrm{~Hz}, 6 \mathrm{H}) .{ }^{13} \mathrm{C} \mathrm{NMR}\left(125 \mathrm{MHz}, \mathrm{CDCl}_{3}\right) \delta 145.9,138.6,133.5,129.0(2 \mathrm{C}), 128.5$ (2C), 110.4, 102.2, $61.1(2 \mathrm{C}), 56.0,34.6,31.5,16.2,15.3$ (2C). Anal calcd for $\mathrm{C}_{17} \mathrm{H}_{26} \mathrm{O}_{4} \mathrm{~S}: \mathrm{C}, 62.55 \%$; H, 8.03\%. Found: C, 62.64\%; H, 7.71\%.

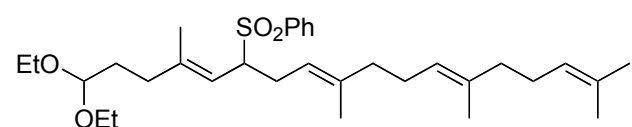

Compound 78. LDA (2.4 mL DIPA, $10.2 \mathrm{~mL}$ of $1.55 \mathrm{M}$-BuLi, $15 \mathrm{~mL}$ THF) was added to a cooled $\left(-78^{\circ} \mathrm{C}\right)$ solution of $\mathbf{5 3}(3.44 \mathrm{~g}, 10.54 \mathrm{mmol})$ in $33 \mathrm{~mL}$ THF and $12 \mathrm{~mL}$ DMPU. The resulting bright orange solution was stirred $30 \mathrm{~min}$ at $-78^{\circ} \mathrm{C}$ at which point farnesyl bromide $(3.0 \mathrm{~mL}, 11.06 \mathrm{mmol})$ was added dropwise. During the addition, the flask was removed from the dry ice bath as need to keep the solution homogeneous. The solution changed from bright orange to a light yellow color during the addition. After the addition, the reaction was stirred an additional $10 \mathrm{~min}$, at which time the reaction was quenched with saturated aqueous ammonium chloride. The resulting aqueous layer was extracted three times with ether. The combined organic layers were dried over magnesium sulfate, and concentrated in vacuo. The resulting oily residue was purified via flash chromatography (silica, ether/petroleum ether, gradient) to yield $5.31 \mathrm{~g} \mathrm{(95 \% )} \mathrm{of} 78$ as a colorless oil. $\mathrm{R}_{\mathrm{f}}=0.63$ in $60 \%$ ether/petroelum ether. IR (neat): $1447,1376,1304$, 1147, $1063 \mathrm{~cm}^{-1} .{ }^{1} \mathrm{H}$ NMR $\left(500 \mathrm{MHz}, \mathrm{CDCl}_{3}\right) \delta 7.82(\mathrm{~m}, 2 \mathrm{H}), 7.60(\mathrm{~m}, 1 \mathrm{H}), 7.49(\mathrm{~m}$, 2H), $5.01(\mathrm{~m}, 3 \mathrm{H}), 4.93(\mathrm{t}, \mathrm{J}=6.8 \mathrm{~Hz}, 1 \mathrm{H}), 4.39(\mathrm{t}, \mathrm{J}=5.6 \mathrm{~Hz}, 1 \mathrm{H}), 3.70(\mathrm{dt}, \mathrm{J}=3.2,10.4$ $\mathrm{Hz}, 1 \mathrm{H}), 3.60(\mathrm{~m}, 2 \mathrm{H}), 3.45(\mathrm{~m}, 2 \mathrm{H}), 2.84(\mathrm{ddd}, \mathrm{J}=12.4,7.2,3.2 \mathrm{~Hz}, 1 \mathrm{H}), 2.32$ (ddd, J = 16.0, 7.2, 3.2 Hz, 1H), 2.04-1.91 (m, 12H), 1.65 (s, 3H), 1.57 (s, 3H), $1.56(\mathrm{~s}, 3 \mathrm{H}), 1.54$ $(\mathrm{s}, 3 \mathrm{H}), 1.18(\mathrm{~s}, 3 \mathrm{H}), 1.18(\mathrm{t}, \mathrm{J}=8.0 \mathrm{~Hz}, 6 \mathrm{H}) .{ }^{13} \mathrm{C} \mathrm{NMR}\left(100 \mathrm{MHz}, \mathrm{CDCl}_{3}\right) \delta 144.7$, 
$138.8,138.1,135.2,133.4,131.3,129.1$ (2C), 128.7 (2C), 124.3, 123.8, 118.5, 117.1, 102.3, 64.8, 61.2, 61.1, 39.7 (2C), 34.7, 31.7, 26.7, 26.6, 26.4, 25.7, 17.7, 16.5, 16.4, $15.9,15.4$ (2C). Anal calcd for $\mathrm{C}_{32} \mathrm{H}_{50} \mathrm{O}_{4} \mathrm{~S}: \mathrm{C}, 72.41 \%$; H, 9.49\%. Found: $\mathrm{C}, 72.60 \%$; H, $9.66 \%$.

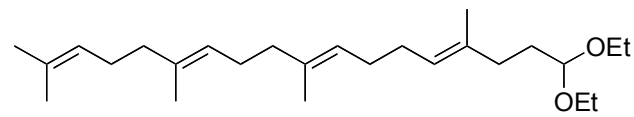

Compound 54. The procedure was performed in analogy to a literature procedure. ${ }^{17} \mathrm{~A}$ slurry of $(\mathrm{Pd}(\pi \text {-allyl }) \mathrm{Cl})_{2}(0.137 \mathrm{~g}, 0.37 \mathrm{mmol})$ and dppp $(0.463 \mathrm{~g}, 1.12 \mathrm{mmol})$ in THF (5 $\mathrm{mL})$ was added to a solution of $78(3.98 \mathrm{~g}, 7.49 \mathrm{mmol})$ in THF $(70 \mathrm{~mL})$ at room temperature. $\mathrm{LiBHEt}_{3}(18.73 \mathrm{~mL}$ of $1.0 \mathrm{M}$ in THF) was then added. The resulting slurry became a yellow solution whose color quickly changed to red and finally to brown. The solution was stirred at room temperature for $45 \mathrm{~min}$, at which time it was quenched carefully at $0^{\circ} \mathrm{C}$ via dropwise addition of saturated aqueous $\mathrm{NH}_{4} \mathrm{Cl}$. The resulting aqueous layer was extracted three times with ether. The combined organic layers were washed with brine, dried over $\mathrm{MgSO}_{4}$, filtered, and concentrated in vacuo. The resulting oily residue was purified via flash chromatography (silica, ether/petroleum ether, gradient) to yield $2.69 \mathrm{~g}(92 \%)$ of 54 as a colorless oil. $R_{\mathrm{f}}=0.67$ in 1:3 ether:petroleum ether, $\mathrm{KMnO}_{4}$ stain. IR (film from $\mathrm{CH}_{2} \mathrm{Cl}_{2}$ ): 1444, 1376, $1199,1132 \mathrm{~cm}^{-1}$. ${ }^{1} \mathrm{H}$ NMR (500 $\left.\mathrm{MHz} \mathrm{CDCl}_{3}\right) \delta$ 5.14-5.06 (m, 4H), $4.44(\mathrm{t}, \mathrm{J}=5.5 \mathrm{~Hz}, 1 \mathrm{H}), 3.62(\mathrm{dq}, \mathrm{J}=9.5,7.0 \mathrm{~Hz}$, $2 \mathrm{H}), 3.47(\mathrm{dq}, \mathrm{J}=9.5,7.0 \mathrm{~Hz}, 2 \mathrm{H}), 2.06-1.94(\mathrm{~m}, 14 \mathrm{H}), 1.70(\mathrm{~m}, 2 \mathrm{H}), 1.66(\mathrm{~s}, 3 \mathrm{H}), 1.58$ $(\mathrm{s}, 12 \mathrm{H}), 1.18(\mathrm{t}, \mathrm{J}=7.0 \mathrm{~Hz}, 6 \mathrm{H}) .{ }^{13} \mathrm{C} \mathrm{NMR}\left(125 \mathrm{MHz}, \mathrm{CDCl}_{3}\right) \delta 135.2,134.9,134.4$, 131.3, 124.5, 124.4, 124.2 (2C), 102.6, 60.9 (2C), 39.7 (2C), 39.7, 34.7, 31.9, 28.2 (2C), 26.7, 26.6, 25.7, 17.7, $16.0(2 \mathrm{C}), 15.36(2 \mathrm{C})$. Anal calcd for $\mathrm{C}_{26} \mathrm{H}_{46} \mathrm{O}_{2}$ : C, 79.94\%; $\mathrm{H}$, $11.87 \%$. Found: C, 79.79\%; H, 11.60\%.

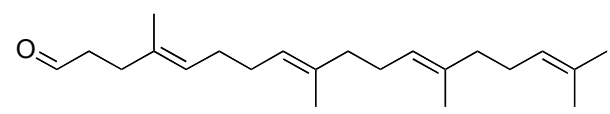

Compound 51. Amberlist- 15 resin $(0.20 \mathrm{~g})$ was added to a room temperature solution of $54(0.50 \mathrm{~g}, 1.28 \mathrm{mmol})$ in acetone $(30 \mathrm{~mL})$ and 10 drops water. The reaction was stirred vigorously under argon for $3 \mathrm{~h}$. The reaction mixture was filtered through a fritted funnel. The filtrate was concentrated in vacuo and purified via flash chromatography (silica, ether/petroleum ether, gradient) to yield $0.369 \mathrm{~g}(91 \%)$ of 51 as a colorless oil. $\mathrm{R}_{\mathrm{f}}=0.5 \mathrm{in}$ 1:14 ether:petroleum ether, double elution, $\mathrm{KMnO}_{4}$ stain. The obtained spectral data [IR, $\left.{ }^{1} \mathrm{H},{ }^{13} \mathrm{C}\right]$ matched the literature values. ${ }^{18}$ IR (neat): $2715,1728,1670,1446 \mathrm{~cm}^{-1} .{ }^{1} \mathrm{H}$ NMR (500 MHz, CDCl $\left.{ }_{3}\right) \delta 9.75(\mathrm{~m}, 1 \mathrm{H}), 5.17(\mathrm{~m}, 1 \mathrm{H}), 5.10(\mathrm{~m}, 3 \mathrm{H}), 2.51(\mathrm{dt}, \mathrm{J}=1.5$, $7.5 \mathrm{~Hz}, 2 \mathrm{H}), 2.31(\mathrm{t}, \mathrm{J}=7.5 \mathrm{~Hz}, 2 \mathrm{H}), 2.09-2.03(\mathrm{~m}, 4 \mathrm{H}), 2.01-1.95(\mathrm{~m}, 8 \mathrm{H}), 1.68(\mathrm{~s}, 3 \mathrm{H})$, $1.61(\mathrm{~s}, 3 \mathrm{H}), 1.60(\mathrm{~s}, 9 \mathrm{H}) .{ }^{13} \mathrm{C} \mathrm{NMR}\left(125 \mathrm{MHz}, \mathrm{CDCl}_{3}\right) \delta$ 202.8, 135.4, 134.9, 133.0, $131.3,125.4,124.3,124.2,124.0,42.1,39.7$ (2C), 31.8, 28.2, 28.0, 26.7, 26.6, 25.7, 17.7, $16.1,16.0(2 \mathrm{C})$. 
$\int_{\text {CotBDPS }}^{\mathrm{Br}}$

Compound 56. The procedure was performed in accordance to a literature procedure. A one-necked $250 \mathrm{~mL}$ round bottom flask equipped with magnetic stir bar and septum was charged with $70 \mathrm{~mL}$ DCM and $14.71 \mathrm{~g}$ anhydrous tetraethylammonium bromide. Note: tetraethylammonium bromide (Aldrich) was first crushed with pestle and azeotropically dried three times from benzene, and then dried under vacuum for 24 hours. The flask was cooled to $0^{\circ} \mathrm{C}$ and weighed. $\mathrm{HBr}$ (gas) was slowly bubbled into the solution (with a vent tube to top of hood). The weight of the flask was checked every 5 min until $7.0 \mathrm{~g}$ had been added. At this point, propargyl alcohol $(4.05 \mathrm{~mL}, 69.6 \mathrm{mmol})$ was added and the flask was removed from the ice bath. The resulting solution was stir at room temperature for $1 \mathrm{~h}$. The solution was then recooled to $0^{\circ} \mathrm{C}$ and poured into a $1000 \mathrm{~mL}$ erlenmeyer flask. The solution was then quenched via slow and dropwise addition of triethylamine $(50 \mathrm{~mL})$. The quenched solution was diluted with $200 \mathrm{~mL}$ of water and $200 \mathrm{~mL}$ of diethyl ether. The resulting organic layer was washed with saturated bicarbonate solution and brine, dried over magnesium sulfate, and concentrated in vacuo. The resulting oily residue was purified via flash chromatography (silica, ether/petroleum ether, gradient) to yield $6.4 \mathrm{~g}(67 \%)$ of vinyl bromide as a colorless oil. $\mathrm{R}_{\mathrm{f}}=0.5$ in $30 \%$ ether/petroleum ether, $\mathrm{KMnO}_{4}$ stain. This vinyl bromide $(4.4 \mathrm{~g}, 32.6 \mathrm{mmol})$ was dissolved in $120 \mathrm{~mL}$ DCM. The flask was cooled to $0^{\circ} \mathrm{C}$ and imidazole $(3.17 \mathrm{~g}, 46.5 \mathrm{mmol})$ and TBDPSCl $(8.7$ $\mathrm{mL}, 33.9 \mathrm{mmol}$ ) were added. The reaction was stirred $20 \mathrm{~min}$ and then quenched with saturated aqueous ammonium chloride solution. The aqueous layer was extracted three times with DCM. The combined organic layers were dried over magnesium sulfate and concentrated in vacuo. The resulting oily residue was purified via flash chromatography (silica, ether/petroleum ether, gradient) to yield $11.1 \mathrm{~g}(91 \%)$ of 56 as a colorless oil. $\mathrm{R}_{\mathrm{f}}=$ 0.81 in $30 \%$ ether/petroleum ether, $\mathrm{KMnO}_{4}$ stain. IR (neat): 1640, 1391, 891, 824, 740 $\mathrm{cm}^{-1} .{ }^{1} \mathrm{H}$ NMR $\left(500 \mathrm{MHz}, \mathrm{CDCl}_{3}\right) \delta 7.74(\mathrm{~d}, \mathrm{~J}=7.0 \mathrm{~Hz}, 4 \mathrm{H}), 7.47(\mathrm{~m}, 6 \mathrm{H}), 6.17(\mathrm{~s}, 1 \mathrm{H})$, $5.64(\mathrm{~s}, 1 \mathrm{H}), 4.31(\mathrm{~s}, 2 \mathrm{H}), 1.15(\mathrm{~s}, 9 \mathrm{H}) .{ }^{13} \mathrm{C} \mathrm{NMR}\left(125 \mathrm{MHz}, \mathrm{CDCl}_{3}\right) \delta 135.4(4 \mathrm{C}), 132.9$ (2C), 131.1, 129.9 (2C), 127.8 (4C), 114.9, 67.8, 26.7 (3C), 19.3. Anal calcd for $\mathrm{C}_{19} \mathrm{H}_{23} \mathrm{OBrSi}$ : C, 60.79\%; H, 6.18\%. Found: C, 60.52\%; H, 5.95\%.

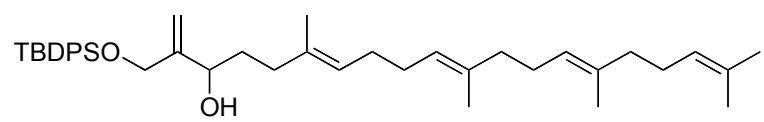

Compound 57. A solution of $51(2.1 \mathrm{~g}, 6.6 \mathrm{mmol})$ and 56 (4.2 g, $13.2 \mathrm{mmol})$ in degassed DMF $(47 \mathrm{~mL})$ was added to a cooled $\left(0^{\circ} \mathrm{C}\right)$ flame dried flask under argon containing $\mathrm{NiCl}_{2}(1.71 \mathrm{~g}, 13.2 \mathrm{mmol})$ and $\mathrm{CrCl}_{2}(4.1 \mathrm{~g}, 33.0 \mathrm{mmol})$. After $5 \mathrm{~min}$, the flask was removed from the ice bath and stirred at room temperature for $2 \mathrm{~h}$, at which time it was cooled to $-10^{\circ} \mathrm{C}$ and quenched with saturated aqueous $\mathrm{NH}_{4} \mathrm{Cl}$ solution. The resulting aqueous layer was extracted four times with ethyl acetate. The combined organic layers were washed with brine to remove the DMF, dried over $\mathrm{MgSO}_{4}$, and concentrated in vacuo. The resulting oily residue was purified via flash chromatography (silica, ether/petroleum ether, gradient) to yield $4.17 \mathrm{~g}(\mathrm{Q})$ of 57 as a colorless oil. $\mathrm{R}_{\mathrm{f}}=0.55$ in $1: 3$ ether:petroleum ether. IR (neat): $3418,1428,1383,1113,823 \mathrm{~cm}^{-1}$. ${ }^{1} \mathrm{H}$ NMR (500 
$\left.\mathrm{MHz}, \mathrm{CDCl}_{3}\right) \delta 7.67(\mathrm{~m}, 4 \mathrm{H}), 7.40(\mathrm{~m}, 6 \mathrm{H}), 5.16(\mathrm{~s}, 1 \mathrm{H}), 5.10(\mathrm{~m}, 5 \mathrm{H}), 4.31(\mathrm{~d}, \mathrm{~J}=13.5$ $\mathrm{Hz}, 1 \mathrm{H}), 4.21(\mathrm{~d}, \mathrm{~J}=13.5 \mathrm{~Hz}, 1 \mathrm{H}), 4.13(\mathrm{t}, \mathrm{J}=5.5 \mathrm{~Hz}, 1 \mathrm{H}), 2.22(\mathrm{~s}, 1 \mathrm{H}), 2.03(\mathrm{~m}, 5 \mathrm{H})$, $1.97(\mathrm{~m}, 9 \mathrm{H}), 1.66(\mathrm{~s}, 3 \mathrm{H}), 1.63(\mathrm{~m}, 2 \mathrm{H}), 1.58(\mathrm{~s}, 9 \mathrm{H}), 1.56(\mathrm{~s}, 3 \mathrm{H}), 1.05(\mathrm{~s}, 9 \mathrm{H}) .{ }^{13} \mathrm{C}$ NMR (125 MHz, $\left.\mathrm{CDCl}_{3}\right) \delta 149.3,135.5$ (4C), 135.2, 134.9, 134.7, 133.1 (2C), 131.3, 129.8 (2C), 127.7 (4C), 124.8, 124.4, 124.2 (2C), 111.3, 73.8, 64.9, 39.7 (2C), 35.8, 33.9, 28.3, 28.2, 26.8 (3C), 26.7, 26.6, 25.7, 19.2, 17.7, 16.0 (3C). Anal calcd for $\mathrm{C}_{41} \mathrm{H}_{60} \mathrm{O}_{2} \mathrm{Si}$ : C, $80.33 \%$; H, 9.87\%. Found: C, $80.12 \%$; H, 9.92\%.

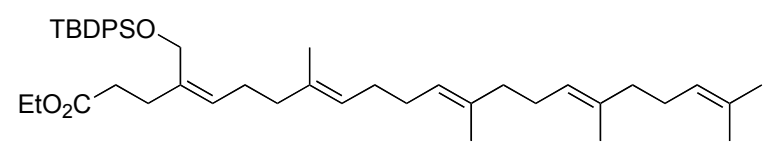

Compound 58. A solution of triethylorthoformate $(9 \mathrm{~mL})$ and $\mathbf{5 7}(2.0 \mathrm{~g}, 3.26 \mathrm{mmol})$ was heated at $100^{\circ} \mathrm{C}$ in sealed flask for $12 \mathrm{~h}$. The reaction was cooled to room temperature and purified directly via flash chromatography (silica, ether/petroleum ether, gradient) to yield $1.96 \mathrm{~g}(88 \%)$ of 58 as a colorless oil. $\mathrm{R}_{\mathrm{f}}=0.64$ in 1:4 ether:petroleum ether, $\mathrm{KMnO}_{4}$ stain. IR (neat): 1738, 1472, 1446, $1428 \mathrm{~cm}^{-1} .{ }^{1} \mathrm{H}$ NMR $\left(500 \mathrm{MHz}, \mathrm{CDCl}_{3}\right) \delta 7.69$ (m, 4H), $7.40(\mathrm{~m}, 6 \mathrm{H}), 5.23(\mathrm{~m}, 1 \mathrm{H}), 5.11(\mathrm{~m}, 3 \mathrm{H}), 5.05(\mathrm{~m}, 1 \mathrm{H}), 4.21(\mathrm{~s}, 2 \mathrm{H}), 4.11(\mathrm{q}, \mathrm{J}=$ $7.0 \mathrm{~Hz}, 2 \mathrm{H}), 2.54(\mathrm{~m}, 2 \mathrm{H}), 2.48(\mathrm{~m}, 2 \mathrm{H}), 2.08(\mathrm{~m}, 5 \mathrm{H}), 1.99(\mathrm{~m}, 9 \mathrm{H}), 1.89(\mathrm{~m}, 2 \mathrm{H}), 1.69$ $(\mathrm{s}, 3 \mathrm{H}), 1.61(\mathrm{~s}, 9 \mathrm{H}), 1.49(\mathrm{~s}, 3 \mathrm{H}), 1.25(\mathrm{t}, \mathrm{J}=7.0 \mathrm{~Hz}, 3 \mathrm{H}), 1.06(\mathrm{~s}, 9 \mathrm{H}) .{ }^{13} \mathrm{C}$ NMR $(125$ $\left.\mathrm{MHz}, \mathrm{CDCl}_{3}\right) \delta 173.4,136.5,135.6(4 \mathrm{C}), 135.1,134.8,134.3,133.6$ (2C), 131.1, 129.6 (2C), 127.6 (4C), 127.1, 124.6, 124.4, 124.2 (2C), 61.2, 60.1, 39.7, 39.6, 33.6, 30.1, 28.2 (2C), 26.8 (4C), 26.7, 26.6, 26.0, 25.7, 19.2, 17.6, 16.0 (2C), 15.9, 14.2. Anal calcd for $\mathrm{C}_{45} \mathrm{H}_{66} \mathrm{O}_{3} \mathrm{Si}: \mathrm{C}, 79.12 \%$; H, 9.74\%. Found: C, 79.38\%; H, 9.60\%.

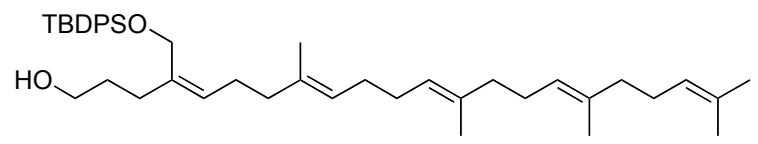

Compound 79. DIBAl-H (5.4 mL of 1.0 $\mathrm{M}$ in hexanes) was added dropwise to a cooled $\left(0^{\circ} \mathrm{C}\right)$ solution of $58(1.06 \mathrm{~g}, 1.55 \mathrm{mmol})$ in THF $(15 \mathrm{~mL})$. The resulting solution was stirred at $0^{\circ} \mathrm{C}$ for $5 \mathrm{~h}$, at which time it was quenched with saturated aqueous Rochelle's salt. The solution was diluted with ether and stirred vigorously at room temperature to break up salts. Once the layers have separated, the aqueous layer was extracted 3 times with ethyl acetate. The combined organic layers were dried over $\mathrm{MgSO}_{4}$ and concentrated in vacuo. The resulting oily residue was purified via flash chromatography (silica, ether/petroleum ether, gradient) to yield $0.9022 \mathrm{~g}(91 \%)$ of 79 as a colorless oil. $\mathrm{R}_{\mathrm{f}}=0.54$ in 1:1 ether:petroleum ether, $\mathrm{KMnO}_{4}$ stain. IR (neat): 3334, 1428, 1383, 1112 , $1063 \mathrm{~cm}^{-1} .{ }^{1} \mathrm{H}$ NMR $\left(500 \mathrm{MHz}, \mathrm{CDCl}_{3}\right) \delta 7.66(\mathrm{~m}, 4 \mathrm{H}), 7.38(\mathrm{~m}, 6 \mathrm{H}), 5.21(\mathrm{~m}, 1 \mathrm{H}), 5.09$ $(\mathrm{m}, 3 \mathrm{H}), 5.03(\mathrm{~m}, 1 \mathrm{H}), 4.17(\mathrm{~s}, 2 \mathrm{H}), 3.62(\mathrm{~m}, 2 \mathrm{H}), 2.24(\mathrm{t}, \mathrm{J}=7.5 \mathrm{~Hz}, 2 \mathrm{H}), 2.04(\mathrm{~m}, 4 \mathrm{H})$, $1.96(\mathrm{~m}, 8 \mathrm{H}), 1.89(\mathrm{~m}, 4 \mathrm{H}), 1.69(\mathrm{~m}, 2 \mathrm{H}), 1.66(\mathrm{~s}, 3 \mathrm{H}), 1.58($ broad s, 9H), $1.47(\mathrm{~s}, 3 \mathrm{H})$, $1.03(\mathrm{~s}, 9 \mathrm{H})$. The hydroxyl proton was too broad to be observed. ${ }^{13} \mathrm{C}$ NMR $(125 \mathrm{MHz}$, $\left.\mathrm{CDCl}_{3}\right) \delta 137.4,135.6(4 \mathrm{C}), 135.1,134.9,134.5,133.6(2 \mathrm{C}), 131.3,129.6(2 \mathrm{C}), 127.7$ (4C), 127.1, 124.6, 124.4, 124.2 (2C), 62.8, 61.3, 39.7 (2C), 31.2, 30.9, 28.2 (2C), 26.8 
(4C), 26.7, 26.6, 25.9, 25.7, 19.2, 17.7, 16.0 (2C), 15.9. Anal calcd for $\mathrm{C}_{43} \mathrm{H}_{64} \mathrm{O}_{2} \mathrm{Si}$ : C, $80.56 \%$; H, 10.06\%. Found: C, 80.38\%; H, 9.96\%.

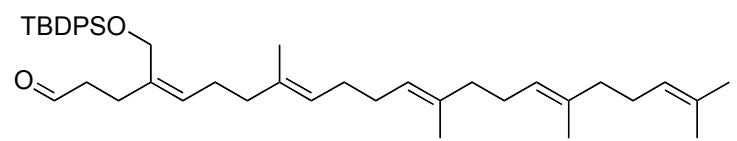

Compound 59. A flask is charged with a solution of $79(0.403 \mathrm{~g}, 0.63 \mathrm{mmol})$ in methylene chloride $(2.0 \mathrm{~mL})$ and pyridine $(0.051 \mathrm{~g}, 0.64 \mathrm{mmol})$. The flask is cooled to $0^{\circ} \mathrm{C}$ at which time Dess-Martin periodinane $(0.325 \mathrm{~g}, 0.767 \mathrm{mmol})$ was added. The resulting slurry was stirred at $0^{\circ} \mathrm{C}$ for $1 \mathrm{~h}$ at which time it was quenched with saturated aqueous sodium bicarbonate and sodium thiosulfate. The resulting aqueous layer was extracted three times with methylene chloride. The combined organic layers were dried over $\mathrm{MgSO}_{4}$, concentrated in vacuo, and purified via flash chromatography (silica, ether/petroleum ether, gradient) to yield $0.328 \mathrm{~g}(82 \%)$ of $\mathbf{5 9}$ as a colorless oil. $\mathrm{R}_{\mathrm{f}}=0.54$ in 1:1 ether:petroleum ether, $\mathrm{KMnO}_{4}$ stain. IR (neat): $2713,1728,1428 \mathrm{~cm}^{-1}$. ${ }^{1} \mathrm{H}$ NMR $\left(500 \mathrm{MHz}, \mathrm{CDCl}_{3}\right) \delta 9.74(\mathrm{t}, \mathrm{J}=1.5 \mathrm{~Hz}, 1 \mathrm{H}), 7.67(\mathrm{~m}, 4 \mathrm{H}), 7.40(\mathrm{~m}, 6 \mathrm{H}), 5.20(\mathrm{~m}, 1 \mathrm{H})$, $5.10(\mathrm{~m}, 3 \mathrm{H}), 5.03(\mathrm{~m}, 1 \mathrm{H}), 4.19(\mathrm{~s}, 2 \mathrm{H}), 2.54(\mathrm{~m}, 4 \mathrm{H}), 2.05(\mathrm{~m}, 4 \mathrm{H}), 1.97(\mathrm{~m}, 8 \mathrm{H}), 1.87$ $(\mathrm{m}, 4 \mathrm{H}), 1.67(\mathrm{~d}, \mathrm{~J}=1.0 \mathrm{~Hz}, 3 \mathrm{H}), 1.58(\mathrm{~m}, 9 \mathrm{H}), 1.47(\mathrm{~d}, \mathrm{~J}=1.0 \mathrm{~Hz}, 3 \mathrm{H}), 1.04(\mathrm{~s}, 9 \mathrm{H}) .{ }^{13} \mathrm{C}$ NMR (125 MHz, $\left.\mathrm{CDCl}_{3}\right) \delta 202.8,136.2,135.6$ (4C), 135.1, 134.9, 134.3, 133.5 (2C), 131.2, 129.7 (2C), 127.7 (4C), 127.4, 124.7, 124.4, 124.2 (2C), 61.2, 42.6, 39.7, 39.6, 28.2 (2C), 27.3, 26.8 (4C), 26.7, 26.6, 25.9, 25.7, 19.2, 17.7, 16.0 (2C), 15.9. Anal calcd for $\mathrm{C}_{43} \mathrm{H}_{62} \mathrm{O}_{2} \mathrm{Si}$ : C, $80.82 \%$; H, 9.78\%. Found: C, 80.66\%; H, 9.71\%.

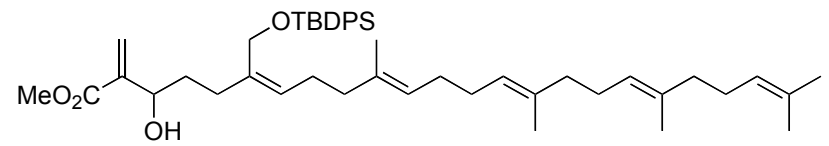

Compound 60. A solution of HMPA $(0.41 \mathrm{~mL}, 2.43 \mathrm{mmol})$ and THF $(4.0 \mathrm{~mL})$ was cooled to $0^{\circ} \mathrm{C}$. DIBAl-H $(0.79 \mathrm{~mL}$ of $1.0 \mathrm{M}$ in hexanes $)$ was added dropwise. The resulting solution was stirred at $0^{\circ} \mathrm{C}$ for $1 \mathrm{~h}$, upon which time, methyl propiolate $(74 \mu \mathrm{L}$, $0.886 \mathrm{mmol}$ ) was added and the resulting solution was stirred for an additional $1.5 \mathrm{~h}$. The solution became a light yellow color. A solution of $59(0.1673 \mathrm{~g}, 0.262 \mathrm{mmol})$ and THF $(1.0 \mathrm{~mL})$ was then added via cannula. After the addition, the solution was warmed to room temperature and stirred for $4 \mathrm{~h}$. The mixture was then cooled to $0^{\circ} \mathrm{C}$ and quenched carefully with $1 \mathrm{~N} \mathrm{HCl}$. The resulting aqueous layer was extracted three times with ether. The combined organic layers were washed with a saturated sodium bicarbonate aqueous solution, dried over $\mathrm{MgSO}_{4}$, and concentrated in vacuo. The resulting oily residue was purified via flash chromatography (silica, ether/petroleum ether, gradient) to yield 0.1564 $\mathrm{g}(82 \%)$ of 60 as a light yellow oil. $\mathrm{R}_{\mathrm{f}}=0.58$ in 1:2 ether:petroleum ether, $\mathrm{KMnO}_{4}$ stain. IR (film from $\mathrm{CH}_{2} \mathrm{Cl}_{2}$ ): 3481, 1721, 1442, $1428 \mathrm{~cm}^{-1} .{ }^{1} \mathrm{H} \mathrm{NMR}\left(500 \mathrm{MHz}, \mathrm{CDCl}_{3}\right) \delta 7.66$ $(\mathrm{m}, 4 \mathrm{H}), 7.39(\mathrm{~m}, 6 \mathrm{H}), 6.22(\mathrm{~d}, \mathrm{~J}=1.0 \mathrm{~Hz}, 1 \mathrm{H}), 5.78(\mathrm{t}, \mathrm{J}=1.5 \mathrm{~Hz}, 1 \mathrm{H}), 5.23(\mathrm{~m}, 1 \mathrm{H})$, $5.07(\mathrm{~m}, 3 \mathrm{H}), 5.03(\mathrm{~m}, 1 \mathrm{H}), 4.40(\mathrm{~m}, 1 \mathrm{H}), 4.20(\mathrm{~d}, \mathrm{~J}=15.0 \mathrm{~Hz}, 1 \mathrm{H}), 4.15(\mathrm{~d}, \mathrm{~J}=15.0 \mathrm{~Hz}$, $1 \mathrm{H}), 3.74(\mathrm{~s}, 3 \mathrm{H}), 2.66(\mathrm{~d}, \mathrm{~J}=8.5 \mathrm{~Hz}, 1 \mathrm{H}), 2.80(\mathrm{~m}, 2 \mathrm{H}), 2.04(\mathrm{~m}, 4 \mathrm{H}), 1.96(\mathrm{~m}, 8 \mathrm{H})$, $1.88(\mathrm{~m}, 4 \mathrm{H}), 1.72(\mathrm{~m}, 2 \mathrm{H}), 1.66(\mathrm{~d}, \mathrm{~J}=1.5 \mathrm{~Hz}, 3 \mathrm{H}), 1.58(\mathrm{~m}, 9 \mathrm{H}), 1.47(\mathrm{~d}, \mathrm{~J}=1.5 \mathrm{~Hz}$, 3H), $1.02(\mathrm{~s}, 9 \mathrm{H}) .{ }^{13} \mathrm{C}$ NMR $\left(125 \mathrm{MHz}, \mathrm{CDCl}_{3}\right) \delta 166.9,142.3,137.3,135.6(4 \mathrm{C}), 135.1$, 
$134.9,134.5,133.6(2 \mathrm{C}), 131.2,129.6(2 \mathrm{C}), 127.6(4 \mathrm{C}), 127.3,125.1,124.5,124.4(2 \mathrm{C})$, 124.2, 71.4, 61.2, 51.8, 39.7 (2C), 34.7, 31.1, 28.2 (2C), 26.8 (4C), 26.7, 26.6, 26.0, 25.7, 19.2, 17.7, $16.0(2 \mathrm{C}), 15.9$. Anal calcd for $\mathrm{C}_{47} \mathrm{H}_{68} \mathrm{O}_{4} \mathrm{Si}: \mathrm{C}, 77.85 \%$; H, 9.45\%. Found: $\mathrm{C}$, $77.96 \% ; \mathrm{H}, 9.37 \%$.

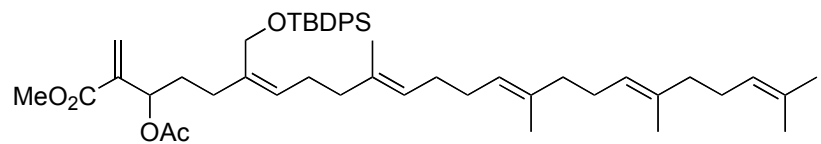

Compound 80. Acetyl chloride $(0.049 \mathrm{~mL}, 0.688 \mathrm{mmol})$ was added to a cooled $\left(0^{\circ} \mathrm{C}\right)$ solution of $60(0.1992 \mathrm{~g}, 0.275 \mathrm{mmol})$ in methylene chloride $(2.8 \mathrm{~mL})$, pyridine $(0.078$ $\mathrm{mL}, 0.961 \mathrm{mmol})$, and DMAP $(0.0168 \mathrm{~g}, 0.138 \mathrm{mmol})$. The solution became an orange color upon addition of the bases, but turned light yellow upon addition of the acetyl chloride. The reaction was stirred at $0^{\circ} \mathrm{C}$ for $2 \mathrm{~h}$, at which time it was quenched with saturated aqueous ammonium chloride. The aqueous layer was extracted three times with methylene chloride. The combined organic layers were dried over $\mathrm{MgSO}_{4}$, concentrated in vacuo, and purified via flash chromatography (silica, ether/petroleum ether, gradient) to yield $0.1975 \mathrm{~g}(94 \%)$ of $\mathbf{8 0}$ as a colorless oil. $\mathrm{R}_{\mathrm{f}}=0.70$ in 1:2 ether:petroleum ether, cerium molybdate stain. IR (film from $\mathrm{CH}_{2} \mathrm{Cl}_{2}$ ): 1750, 1720, 1429, 1290, 1112, 1071, 1045, $703 \mathrm{~cm}^{-1} .{ }^{1} \mathrm{H}$ NMR $\left(400 \mathrm{MHz}, \mathrm{CDCl}_{3}\right) \delta 7.65(\mathrm{~m}, 4 \mathrm{H}), 7.39(\mathrm{~m}, 6 \mathrm{H}), 6.27(\mathrm{~s}, 1 \mathrm{H})$, $5.74(\mathrm{~s}, 1 \mathrm{H}), 5.59(\mathrm{dd}, \mathrm{J}=7.6,4.0 \mathrm{~Hz}, 1 \mathrm{H}), 5.16(\mathrm{~m}, 1 \mathrm{H}), 5.10(\mathrm{~m}, 3 \mathrm{H}), 5.02(\mathrm{~m}, 1 \mathrm{H})$, $4.19(\mathrm{~d}, \mathrm{~J}=12.0 \mathrm{~Hz}, 1 \mathrm{H}), 4.15(\mathrm{~d}, \mathrm{~J}=12.0 \mathrm{~Hz}, 1 \mathrm{H}), 2.73(\mathrm{~s}, 3 \mathrm{H}), 2.31(\mathrm{~m}, 1 \mathrm{H}), 2.17(\mathrm{~m}$, $1 \mathrm{H}), 2.03(\mathrm{~s}, 3 \mathrm{H}), 2.04(\mathrm{~m}, 4 \mathrm{H}), 1.95(\mathrm{~m}, 8 \mathrm{H}), 1.85(\mathrm{~m}, 4 \mathrm{H}), 1.80(\mathrm{~m}, 2 \mathrm{H}), 1.66(\mathrm{~d}, \mathrm{~J}=$ $0.8 \mathrm{~Hz}, 3 \mathrm{H}), 1.57(\mathrm{~m}, 9 \mathrm{H}), 1.46(\mathrm{~s}, 3 \mathrm{H}), 1.02(\mathrm{~s}, 9 \mathrm{H}) .{ }^{13} \mathrm{C} \mathrm{NMR}\left(100 \mathrm{MHz}, \mathrm{CDCl}_{3}\right) \delta$ $169.9,165.7,140.2,137.0,135.6$ (4C), 135.1, 134.9, 134.6, 133.7, 133.6 (2C), 131.3, 129.6 (2C), 127.6 (4C), 126.7, 125.1, 124.5, 124.4, 124.2, 71.7, 61.0, 51.9, 39.7 (2C), 33.0 (2C), 30.4, 28.2 (2C), 26.8 (3C), 26.7, 26.6, 26.0, 25.7, 21.1, 19.2, 17.7, 16.0 (2C), 15.9. This compound was fully characterized after conversion to 61 .

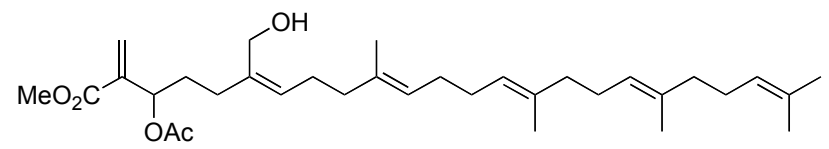

Compound 61. A stock solution of buffered TBAF (1.0 mL of $0.1 \mathrm{M}$ TBAF, $0.1 \mathrm{M}$ $\mathrm{AcOH}$, and $0.5 \mathrm{M}$ water in DMF) was added to a solution of $\mathbf{8 0}(0.055 \mathrm{~g}, 0.0716 \mathrm{mmol})$ in $0.3 \mathrm{~mL}$ of DMF under argon. The resulting solution was stirred for $20 \mathrm{~h}$ at which time it was quenched with saturated aqueous sodium bicarbonate solution and water. The resulting aqueous layer was extracted three times with ether. The combined organic layers were washed with water and brine, dried over $\mathrm{MgSO}_{4}$, and concentrate in vacuo. The resulting oily residue was purified via flash chromatography (silica, ether/petroleum ether, gradient) to yield $0.028 \mathrm{~g}(75 \%)$ of 61 as a light yellow oil. $\mathrm{R}_{\mathrm{f}}=0.17$ in $1: 1$ ether:petroleum ether, cerium molybdate stain. IR (film from $\mathrm{CDCl}_{3}$ ): $3532,1746,1725$, 1440, $1370 \mathrm{~cm}^{-1} .{ }^{1} \mathrm{H}$ NMR $\left(500 \mathrm{MHz}, \mathrm{CDCl}_{3}\right) \delta 6.26(\mathrm{~s}, 1 \mathrm{H}), 5.75(\mathrm{~s}, 1 \mathrm{H}), 5.56(\mathrm{dd}, \mathrm{J}=$ 8.5, $4.0 \mathrm{~Hz}, 1 \mathrm{H}), 5.28$ (t, J = 7.5 Hz, 1H), $5.08(\mathrm{~m}, 4 \mathrm{H}), 4.10(\mathrm{~s}, 2 \mathrm{H}), 3.74(\mathrm{~s}, 3 \mathrm{H}), 2.15$ $(\mathrm{m}, 4 \mathrm{H}), 2.06(\mathrm{~s}, 3 \mathrm{H}), 2.04(\mathrm{~m}, 5 \mathrm{H}), 1.98(\mathrm{~m}, 11 \mathrm{H}), 1.66(\mathrm{~s}, 3 \mathrm{H}), 1.57$ (broad s, 12H). The hydroxyl proton was too broad to be observed. ${ }^{13} \mathrm{C}$ NMR $\left(125 \mathrm{MHz}, \mathrm{CDCl}_{3}\right) \delta 169.9$, 
$165.8,139.9,137.0,135.2,134.9,134.5,131.3,129.5,125.2,124.9,124.4,124.2,124.1$, 71.3, 60.2, 52.0, 39.8, 39.7, 32.8 (2C), 31.2, 28.2 (2C), 26.7, 26.6, 26.3, 25.7, 21.0, 17.7, 16.0 (2C) 15.9. Anal calcd for $\mathrm{C}_{33} \mathrm{H}_{52} \mathrm{O}_{5}$ : C, 74.96\%; H, 9.91\%. Found: C, $74.70 \%$; H, $10.05 \%$.

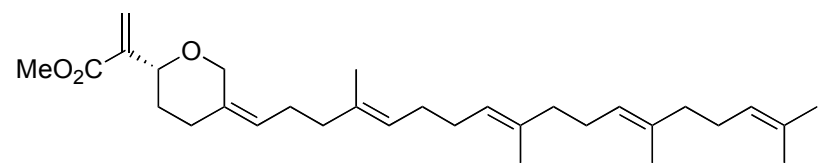

Compound 62. A solution of $(\mathrm{Pd}(\pi-\text { allyl }) \mathrm{Cl})_{2}(0.00044 \mathrm{~g}, 0.0012 \mathrm{mmol})$ and $(R, R)-\mathbf{L}-\mathbf{3}$ $(0.0028 \mathrm{~g}, 0.0036 \mathrm{mmol})$ in $0.3 \mathrm{~mL}$ degassed dioxane was added via cannula to a solution of 61 (0.03 g, $0.06 \mathrm{mmol})$, and tetrahexylammonium chloride $(0.007 \mathrm{~g}, 0.018 \mathrm{mmol})$ in degassed dioxane $(0.3 \mathrm{~mL})$. The reaction was stirred at $80^{\circ} \mathrm{C}$ under argon for $17 \mathrm{~h}$. The resulting light yellow solution was purified directly via flash chromatography (silica, ether/petroleum ether, gradient) to yield $0.014 \mathrm{~g}(50 \%)$ of $62 .[\alpha]_{\mathrm{D}}+20.38(\mathrm{c}=0.42$, $\mathrm{CH}_{2} \mathrm{Cl}_{2}$ ) for $91 \%$ ee as determined via chiral HPLC analysis. (Chiralcel AD column, 400:1 heptane: $\mathrm{PrOH}$, flow rate $=0.5 \mathrm{~mL} / \mathrm{min}, 230 \mathrm{~nm}, \mathrm{t}_{\mathrm{r}}: 13.64$ (minor), 17.22 (major)). The obtained spectral data $\left[\mathrm{IR},{ }^{1} \mathrm{H},{ }^{13} \mathrm{C}\right]$ matched the literature values. ${ }^{19}$ IR (film from $\left.\mathrm{CDCl}_{3}\right): 2918,2850,1722,1633,1439,955,819 \mathrm{~cm}^{-1} .{ }^{1} \mathrm{H} \mathrm{NMR}\left(500 \mathrm{MHz}, \mathrm{CDCl}_{3}\right) \delta$ $6.23(\mathrm{t}, \mathrm{J}=1.5 \mathrm{~Hz}, 1 \mathrm{H}), 5.88(\mathrm{t}, \mathrm{J}=1.5 \mathrm{~Hz}, 1 \mathrm{H}), 5.18(\mathrm{t}, \mathrm{J}=7.0 \mathrm{~Hz}, 1 \mathrm{H}), 5.12-5.06(\mathrm{~m}$, 4H), $4.67(\mathrm{~d}, \mathrm{~J}=12.5 \mathrm{~Hz}, 1 \mathrm{H}), 4.31(\mathrm{~d}, \mathrm{~J}=11.5 \mathrm{~Hz}, 1 \mathrm{H}), 3.86(\mathrm{~d}, \mathrm{~J}=12.5 \mathrm{~Hz}, 1 \mathrm{H}), 3.75$ (s, 3H), 2.36-2.27 (m, 2H), 2.15-1.94 (m, 17H), $1.66(\mathrm{~d}, \mathrm{~J}=1.5 \mathrm{~Hz}, 3 \mathrm{H}), 1.58(\mathrm{~s}, 3 \mathrm{H})$, $1.57(\mathrm{~s}, 9 \mathrm{H}), 1.33(\mathrm{~m}, 1 \mathrm{H}) .{ }^{13} \mathrm{C} \mathrm{NMR}\left(125 \mathrm{MHz}, \mathrm{CDCl}_{3}\right) \delta$ 166.3, 141.5, 135.2, 134.9, $134.4,132.9,131.3,124.8,124.6,124.5,124.4,124.2$ (2C), 75.4, 67.1, 51.8, 39.8, 39.7, $33.9,33.0,29.7,28.2(2 \mathrm{C}), 26.7,26.6,25.7,25.6,17.7,16.1,16.0(2 \mathrm{C})$.

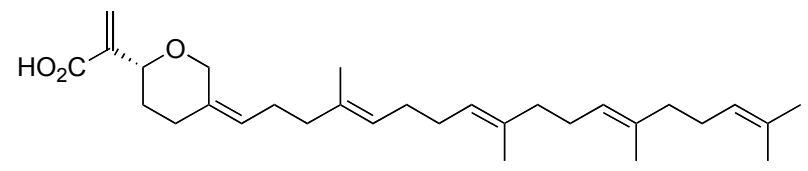

(+)-Hippospongic acid A (Compound 35). The procedure was performed in accordance with a literature procedure. ${ }^{19} \mathrm{LiOH}(14 \mathrm{mg}$ in $0.11 \mathrm{~mL}$ water) was added dropwise over 1 hour via syringe pump to a cooled $\left(0^{\circ} \mathrm{C}\right)$ solution of $62(0.0055 \mathrm{~g}, 0.0117 \mathrm{mmol})$ in THF $(0.11 \mathrm{~mL})$. After the addition, the ice bath was removed and the solution was warmed to room temperature and stirred for $20 \mathrm{~h}$. Upon completion, the reaction mixture was acidified with $1 \mathrm{M} \mathrm{HCl}$ and diluted with ether. The aqueous layer was separated and extracted three times with ether. The combined organic layers were washed with brine, dried over anhydrous magnesium sulfate, filtered, and concentrated in vacuo. The resulting oily residue was purified via flash chromatography on deactivated silica $(10 \%$ wt water on silica gel) with hexane:EtOAc $4: 1$ to yield $5.2 \mathrm{mg}(98 \%$ yield) of $(+)$ hippospongic acid $\mathrm{A}(\mathbf{3 5})$ as a colorless oil. The obtained spectral data $\left[\mathrm{IR},{ }^{1} \mathrm{H},{ }^{13} \mathrm{C},[\alpha]_{\mathrm{D}}\right]$ matched the literature values. ${ }^{19,20}$ Furthermore, the obtained spectral data matched the authentic spectra of natural (+)-hippospongic acid A. ${ }^{21}$ Natural (+)-hippospongic acid A: 
$[\alpha]_{\mathrm{D}}+37.0\left(\mathrm{c}=0.22, \mathrm{CHCl}_{3}\right){ }^{22}$ Our synthetic hippospongic acid $\mathrm{A}:[\alpha]_{\mathrm{D}}+37.4(\mathrm{c}=0.52$, $\mathrm{CHCl}_{3}$ ), for $91 \%$ ee. IR (film from $\mathrm{CHCl}_{3}$ ): 2922, 2852, 1694, 1435, 1288, 1083, 1046 $\mathrm{cm}^{-1} .{ }^{1} \mathrm{H}$ NMR $\left(400 \mathrm{MHz}, \mathrm{CDCl}_{3}\right) \delta 6.35(\mathrm{~s}, 1 \mathrm{H}), 5.89(\mathrm{t}, \mathrm{J}=1.2 \mathrm{~Hz}, 1 \mathrm{H}), 5.22(\mathrm{t}, \mathrm{J}=1.5$ $\mathrm{Hz}, 1 \mathrm{H}), 5.12-5.05(\mathrm{~m}, 4 \mathrm{H}), 4.71(\mathrm{~d}, \mathrm{~J}=12.8 \mathrm{~Hz}, 1 \mathrm{H}), 4.30(\mathrm{~d}, \mathrm{~J}=11.2 \mathrm{~Hz}, 1 \mathrm{H}), 3.89$ (d, $\mathrm{J}=12.8 \mathrm{~Hz}, 1 \mathrm{H}), 2.33(\mathrm{~m}, 1 \mathrm{H}), 2.10-1.93(\mathrm{~m}, 18 \mathrm{H}), 1.66(\mathrm{t}, \mathrm{J}=1.2 \mathrm{~Hz}, 3 \mathrm{H}), 1.58(\mathrm{~s}$, $12 \mathrm{H}), 1.49(\mathrm{~m}, 1 \mathrm{H})$, carboxylic acid peak not seen. ${ }^{13} \mathrm{C} \mathrm{NMR}\left(125 \mathrm{MHz}, \mathrm{CDCl}_{3}\right) \delta 167.7$, $140.0,135.2$, 134.9, 134.3, 132.0, 131.3, 127.1, 125.4, 124.9, 124.4, 124.2 (2C), 76.3, 67.2, 39.7 (2C), 33.4, 32.8, 29.7, 28.7, 28.2, 26.7, 26.6, 25.7, 25.6, 17.7, 16.1, 16.0 (2C). HRMS $\left(\mathrm{M}^{+}\right) \mathrm{C}_{30} \mathrm{H}_{46} \mathrm{O}_{3}$. Calculated: 454.3447. Found 454.3455.

\footnotetext{
${ }^{1}$ Tasuno, Y.; Toshida, T.; Otsuka, S. Inorg. Syn. 1979, 19, 220.

${ }^{2}$ Ukai, T.; Kawazura, H.; Iishii, Y.; Bonnett, J; Ibers, J. A. J. Organomet. Chem.1974, 65, 263.

3 (a) Trost, B. M.; Van Vranken, D. L. Angew. Chem. Int. Ed.. 1992, 31, 228. (b). Trost, B. M.; Van Vranken, D. L. J. Am. Chem. Soc. 1992, 114, 9327.

${ }^{4}$ Still, W. C.; Kahn, M.; Mitra, J. A. J. Org. Chem. 1978, 48, 2923.

5 Drewes, S. E.; Emslie, N. D. J. Chem. Soc., Perkin Trans.I 1982, 2079.

${ }^{6}$ Tsui, H. C. Final Report, Stanford University, 2001.

${ }^{7}$ Trost, B. M.; Tsui, H. C.; Toste, F. D. J. Am. Chem. Soc. 2000, 122, 3534.

${ }^{8}$ a) Hosokawa, T.; Miyagi, S.; Murahashi, S.-I.; Sonoda, A J. Org. Chem. 1978, 43, 2752. b) Visser, M. S.; Harrity, J. P. A.; Hoveyda, A. H. J. Am. Chem. Soc. 1996, 118, 3779.

${ }^{9}$ Trost, B. M.; Toste, F. D. J. Am. Chem. Soc. 1998, $120,815$.

${ }^{10}$ Villieras, J.; Rambaud, M.; Graff, M. Synth. Commun. 1986, 16, 149.

${ }^{11}$ Trost, B. M.; Belletire, J. L.; Godleski, S.; McDugal, P. G.; Balkovec, J. M.; Baldwin, J. J.; Chirsty, M. E.; Ponticello, G. S.; Viraga, S. L.; Springer, J. P. J. Org. Chem. 1986, 51, 2370.

12 Trost, B. M.; Toste, F. D. J. Am. Chem. Soc. 2000, 122, 11262.

${ }^{13}$ Corey, E. J.; Achiwa, K.; Katzenellenbogen, J. A. J. Am. Chem. Soc. 1969, 91, 4318.

14 Tago, K.; Masami, A.; Kogen, H. J. Chem. Soc. Perkin Trans. 1 2000, 2073.

${ }^{15}$ Kocienski, P. J.; Brown, R. C. D.; Pommier, A.; Procter, M.; Schmidt, B. J. Chem. Soc. Perkin Trans. 1 1998, 9.

${ }^{16}$ Dodd, D. S.; Oeschlager, A. C. J. Org. Chem. 1992, 7226.

${ }^{17}$ Journal, E. K.; Schmalz, H. G. Synthesis 1997, 2, 202.

${ }^{18}$ Tokumasu, M.; Ando, H.; Hiraha, Y.; Kojima, S.; Ohkata, K. J. Chem. Soc. Perkin Trans. I 1999, 489.

${ }^{19}$ Hiraga, Y.; Ago, M.; Tokumasu, M.; Kaku, K.; Ohkata, K. Aust. J. Chem. 2000, 53, 909.

${ }^{20}{ }^{20}$ Hioki, H; Ooi, H.; Hamano, M; Mimura, Y; Yoshio, S; Kodama, M; Ohta, S; Yanai, M; Ikegami, S. Tetrahedron 2001, 57, 1235.

${ }^{21}$ We thank Professor Shinji Ohta of the Nagahama Institute of Bio-science and Technology for providing copies of the original spectra of (+)-hippospongic acid A.

22 Ohta, S.; Unoo, M.; Tokumasu, M.; Hiraga, Y.; Ikegami, S. Tetrahedron Lett. 1996, 37, 7765.
} 